\title{
密度泛函活性理论中的信息论方法
}

\author{
刘述斌* \\ (Research Computing Center, University of North Carolina, Chapel Hill, North Carolina 27599-3420, USA)
}

\begin{abstract}
摘要: 密度泛函活性理论(DFRT)运用简单的密度泛函探讨和定量化分子的反应活性, 是近来发展起来的一 个关于分子活性理论的新方法。在新近的文献中, 这样的简单密度泛函的例子包括香农熵, 费舍尔信息以及 其它来自信息论中的密度泛函。本文综述了DFRT信息论方法的原理, 包括物理信息极小原理、最小信息增 益原理和信息守恒原理。总结了DFRT信息论方法在电子密度、形态密度和分子中的原子三种表述下的理论 框架。此外, 还介绍了运用信息论方法在定量描述空间位阻效应、亲电性、亲核性和区域选择性中的突出应 用, 以及对亲电芳香取代反应的邻对间位取代效应的起源和本质提供的一个全新诠释。最后简要地展望了该 领域的几个可能的未来发展方向。
\end{abstract}

关键词：香农熵；费舍尔信息；密度泛函理论反应；立体效应；亲电性；亲核性；区域选择性； 邻对间位取代效应

中图分类号: 0641

\section{Information-Theoretic Approach in Density Functional Reactivity Theory}

\author{
LIU Shu-Bin* \\ (Research Computing Center, University of North Carolina, Chapel Hill, North Carolina 27599-3420, USA)
}

\begin{abstract}
Density functional reactivity theory (DFRT) is a recent endeavor to appreciate and quantify molecular reactivity with simple density functionals. Examples of such density functionals recently investigated in the literature included Shannon entropy, Fisher information, and other quantities from information theory. This review presents an overview on the principles of the information-theoretic approach in DFRT, including the extreme physical information principle, minimum information gain principle, and information conservation principle. Three representations of this approach with electron density, shape function, and atoms-in-molecules are also summarized. Moreover, their applications in quantifying steric effect, electrophilicity, nucleophilicity, and regioselectivity are highlighted, so are the recent results in a completely new understanding about the nature and origin of ortho/para and meta group directing phenomena in electrophilic aromatic substitution reactions. A brief outlook of a few possible future developments is discussed at the end.
\end{abstract}

Key Words: Shannon entropy; Fisher information; Density functional reactivity theory; Steric effect; Electrophilicity; Nucleophilicity; Regioselectivity; Ortho/para and meta group directing effect

\section{Introduction}

Density functional theory (DFT) ${ }^{1}$ as an alternative approach to solve the Schrodinger equation for the calculation of electronic structures and properties for molecules and solids has en- joyed tremendous success in the past few decades. It is theoretically rigorous, conceptually attractive, and computationally efficient. Its complete discard of orbitals in the mind of its original inventors is even more enthralling. This is not only because

Received: October 2, 2015; Revised: October 29, 2015; Published on Web: October 30, 2015.

${ }^{*}$ Corresponding author. Email: shubin@email.unc.edu; Tel: +1-919-962-4032

CEditorial office of Acta Physico-Chimica Sinica 
of the complex nature of the single-particle and total wave functions but also the fact that the concept of atomic and molecular orbitals introduced as artifacts is the most crude approximation in the wave function theory. Although Kohn-Sham orbitals were later employed for the practical implementation of DFT, the rigor and effort of developing such a theoretical framework without the use of orbitals have been lingering over people's minds for quite some time, and are still driving a number of people moving towards that direction. Such a practice in DFT is called the orbital-free DFT (OF-DFT) in the literature ${ }^{2-4}$.

Closely related to this endeavor is the application of DFT to chemical reactivity theory in chemistry, where the mainstream for over several decades has been to make use of molecular orbitals and their related properties to predict and rationalize molecular reactivity ${ }^{5-7}$. Examples include Fukui's frontier molecular orbital (FMO) $)^{8}$ theory and the Woodward-Hoffmann's rules through the conservation of the orbital symmetry ${ }^{9}$. Nevertheless, according to DFT, the electron density of a molecular system alone should suffice in determining all its properties in the ground state, including its structure and reactivity properties ${ }^{1}$. Is it possible to recast the reactivity theory with molecular orbitals in the language of the electron density without scarifying the rigor and generality? More importantly, is it possible to expand the scope of our present orbital-based understanding about molecular reactivity with the introduction of descriptors from the electron density and its associated quantities?

The answer to both of the above questions is yes. At least, we believe they should be. Pioneered by its inventor, Robert G. Parr of the University of North Carolina at Chapel Hill, the first such a theoretical framework is the conceptual density functional theory $(\mathrm{CDFT})^{5-7}$, also called chemical DFT, reactivity DFT, among others. It regards the chemical reactivity of a molecular system as the responses to the changes in the number of electrons, external potential, or others, which can be simulated by the perturbation expansion using a Taylor series of the total energy. CDFT interprets these processes by conceptualizing the first and second order derivatives appearing in the Taylor expansion in terms of chemical insights. For that, various reactivity descriptors such as electronegativity, hardness/softness, Fukui function, dual descriptor, etc. ${ }^{5-7}$, have been introduced and linked to relevant chemical meanings in conventional chemical language. They not only rediscovered Fukui's FMO theory ${ }^{1,5-7}$, but also explained the validity of the WoodwardHoffmann rules without revoking orbitals and their symmetry. ${ }^{10}$

The approach of employing the first and second order derivatives in explaining molecular reactivity, however, does not really grasp the essence of DFT. That is, it does not directly employing electron density and its related quantities for the purpose. In retrospect, density related quantities have been extensively utilized by Bader ${ }^{11}$ and others ${ }^{12}$ in categorizing and characterizing chemical bonds and weak interactions. Nevertheless, no systematic effort is seen until recently. There is a recent effort in the literature, trying to establish a chemical reactivity frame- work using functionals based purely on the electron density and its related quantities. Such a practice is now called DFRT (density functional reactivity theory), to differentiate from the original work by Parr and others on CDFT (conceptual density functional theory). To the best knowledge of the present author, the first work along this line is the seminal paper in 2000 by Nalewajski and Parr ${ }^{13}$, who unveiled that minimizing the information gain (also called Kullback-Leibler divergence) leads to the "stockholder partition" of the electron density, i.e., the so-called Hirshfeld charge ${ }^{14}$. In 2007, we proposed to use the Weizsäcker kinetic energy functional as a quantification for the steric effect ${ }^{15}$. Numerous applications have followed since then. Very recently, based on the work by Nalewajski and Parr, reliable predictions of electrophilicity, nucleophilicity and regioselectivity using the Hirshfeld charge and information gain have been reported ${ }^{16-22}$. These novel developments share a couple of common points. On the one hand, they all make use of density functionals, not just density, its gradient or Laplacian used in Bader's AIM (atoms in molecules) theory ${ }^{11}$ as reactivity descriptors. These reactivity related density functionals, on the other hand, all fall into the same umbrella of the informationtheoretic approach, which coincidently has seen tremendous developments in recent years from the physics viewpoin $\mathrm{t}^{23-28}$.

In this review, we outline principles of the information-theoretic approach as well as its recent developments from the chemical reactivity perspective. This is an emerging field so it is impossible to be comprehensive in the literature review. Rather, we focus more on the recent progresses by the present author and coworkers, and, for the benefit of readers and potential researchers, provide personal outlooks for its possible future development directions.

\section{Conceptual DFT and DFRT}

In Conceptual $\mathrm{DFT}^{1,5-7}$, the starting point is the conjecture that any ground-state property of an electronic system such as the total electronic energy, $E$, can be sufficiently described by two variables, the total electron number $N$ and the external potential $v(\boldsymbol{r}), E \equiv E[N, v(\boldsymbol{r})]$. When an attacking agent comes across leading to the changes in both $N$ and $v(\boldsymbol{r}), \Delta N$ and $\Delta v(\boldsymbol{r})$, the subsequent change in the total energy, $\Delta E$, can be approximated by the Taylor expansion up to the second order of the following formula ${ }^{1,5-7}$

$$
\begin{aligned}
\Delta E \equiv & E[N+\Delta N, v(\boldsymbol{r})+\Delta v(\boldsymbol{r})]-E[N, v(\boldsymbol{r})] \\
\approx & \left(\frac{\partial E}{\partial N}\right)_{v} \Delta N+\int\left(\frac{\delta E}{\delta v(\boldsymbol{r})}\right)_{N} \delta v(\boldsymbol{r}) \mathrm{d} \boldsymbol{r}+ \\
& \frac{1}{2 !}\left\{\left(\frac{\partial^{2} E}{\partial N^{2}}\right)_{v} \Delta N^{2}+2 \int\left(\frac{\partial}{\partial N}\left(\frac{\delta E}{\delta v(\boldsymbol{r})}\right)_{N}\right)_{v} .\right. \\
& \left.\Delta N \delta v(\boldsymbol{r}) \mathrm{d} \boldsymbol{r}+\iint\left(\frac{\delta^{2} E}{\delta v^{2}(\boldsymbol{r})}\right)_{N} \delta v(\boldsymbol{r}) \delta v\left(\boldsymbol{r}^{\prime}\right) \mathrm{d} \boldsymbol{r} \mathrm{d} \boldsymbol{r}^{\prime}\right\}
\end{aligned}
$$

where $(\partial E / \partial N)_{v}$ and $[\delta E / \delta v(\boldsymbol{r})]_{N}$ are the first-order partial derivatives of the total energy with respect to $N$ and $v(\boldsymbol{r})$ with $v(\boldsymbol{r})$ and $N$ fixed, respectively, $\left(\partial^{2} E / \partial N^{2}\right)_{v}$ and $\left[\delta^{2} E / \delta v^{2}(\boldsymbol{r})\right]_{N}$ are the 
corresponding second-order terms, and $\left[\partial / \partial N(\delta E / \delta v)_{N}\right]_{v}$ is the second-order cross term.

What conceptual DFT has accomplished over the last few decades was to make sense of these derivatives in terms of chemical concepts. For instance, $(\partial E / \partial N)_{v}$ is nothing but the widely used concept of electronegativity in chemistry. One of the second-order term, $\left(\partial^{2} E / \partial N^{2}\right)_{v}$ was defined as global hardness, $\eta$, because it is closely related to the principle of hard and soft acid and base (HSAB). More importantly, the second-order cross term, $\left[\partial / \partial N(\delta E / \delta v)_{N}\right]_{v}$ was named as the Fukui function, $f(\boldsymbol{r})$, by Parr and Yang ${ }^{29}$, because it recovers the frontier orbital results from Fukui's FMO theory as a special case. A few reviews and treatises on the subject are available in the literature ${ }^{1,5-7}$. Readers are referred to those references for more details. Recent developments include examinations of third-order terms of the above Tayler series. The most prominent work is the dual descriptor by Morell, Grand, and Toro-Labbé in $2005^{30}$,

$$
\begin{aligned}
f^{(2)}(\boldsymbol{r}) & =\left(\frac{\partial^{2}}{\partial^{2} N}\left(\frac{\delta E}{\delta v(\boldsymbol{r})}\right)_{N}\right)_{v}=\left(\frac{\delta}{\delta v(\boldsymbol{r})}\left(\frac{\partial^{2} E}{\partial^{2} N}\right)_{v}\right)_{N} \\
& =\left(\frac{\partial f(\boldsymbol{r})}{\partial N}\right)_{v}=\left(\frac{\delta \eta}{\delta v(\boldsymbol{r})}\right)_{N}
\end{aligned}
$$

This quantity can be used to explain the validity of the WoodwardHoffmann rules, which are often believed that molecular orbitals and their symmetry play the essential role and thus densitybased indices are unable to interpret their origin. The recent work using the dual descriptor clearly demonstrated that orbital and symmetry are not mandatory to interpret the validity of these rules ${ }^{10}$. We recently applied these ideas in molecular acidity/basicity study ${ }^{31-35}$ and in predicting mechanisms for protoncoupled electron transfer reactions $\mathrm{s}^{36,37}$.

While CDFT has been with us for about thirty years with lots of insights provided, establishments accomplished, and progresses and developments still continuing ${ }^{1,5-7}$, there are apparent discrepancies between theory and reality. It is still far from being accepted by the chemical community as the mainstream of chemical reactivity theory. There are ongoing controversies and debates about the general validity of the principles from this theory such as maximum hardness principle and minimum electrophilicity principle ${ }^{38-42}$. Its general applicability and prediction robustness are often questioned.

There is a recent effort in the literature in applying DFT to examine and rationalize molecular reactivity in a vastly different manner. In this case, instead of employing the energy derivatives introduced above, density functionals themselves are directly used to quantify reactivity properties for molecules such as the charge distribution ${ }^{13,43-45}$, steric effect ${ }^{15,46-58}$, and molecular electrophilicity/ nucleophilicity ${ }^{16-22}$. This effort is similar to the conventional undertaking in DFT, where one tries to find more accurate and better behaved density functionals to approximate energy components such as the exchange-correlation energy and non-interacting kinetic energy. What the new approach is trying to establish is to use density functionals to provide new insights for reactivity properties and quantify them if possible.
This very idea of employing density functionals to quantify molecular reactivity is indeed akin to and consistent with the original spirit of the DFT inventors. That is, since the electron density alone should suffice in determining all properties in the ground state, any property of a molecule could be expressed as a functional of the electron density. Since this approach deviate so much from the original idea of CDFT, we call this new approach DFRT, abbreviated from density functional reactivity theory. To be more specific, DFRT stands for a chemical reactivity theory in DFT where molecular reactivity properties are appreciated and quantified by density functionals.

\section{Information-theoretic approach}

\subsection{Electron density representation}

Information theory developed by Shannon ${ }^{59}$ and others as a branch of applied mathematics, electrical engineering, and computer science is involved in the quantification of information, which is often a probability distribution function. Since the electron density is a continuous probability distribution function, as early as in the year of 1980, information theory has been applied to DFT to study atoms and molecules ${ }^{60-62}$. We call this particular category of work the information-theoretic approach.

A key measure of information is entropy, quantifying the uncertainty involved in predicting the value of the distribution function. Shannon entropy is the first such a measure widely used in the literature, which reads ${ }^{59}$

$$
S_{\mathrm{S}}=-\int \rho(\boldsymbol{r}) \ln \rho(\boldsymbol{r}) \mathrm{d} \boldsymbol{r}=\int s_{\mathrm{S}}(\boldsymbol{r}) \mathrm{d} \boldsymbol{r}
$$

where $s_{\mathrm{S}}(\boldsymbol{r})$ is the Shannon entropy density and $\rho(\boldsymbol{r})$ is the total electron density, satisfying the following condition in relation to the total number of electrons, $N$, of the system,

$$
\int \rho(\boldsymbol{r}) \mathrm{d} \boldsymbol{r}=N
$$

Shannon entropy measures the spatial delocalization of the electronic density. The second important measure in information theory is the Fisher information, $I_{\mathrm{F}}$, defined as follows ${ }^{63}$

$$
I_{\mathrm{F}} \equiv \int i_{\mathrm{F}}(\boldsymbol{r}) \mathrm{d} \boldsymbol{r}=\int \frac{|\nabla \rho(\boldsymbol{r})|^{2}}{\rho(\boldsymbol{r})} \mathrm{d} \boldsymbol{r}
$$

which is a gauge of the sharpness or concentration of the electron density distribution. In Eq.(5), $i_{\mathrm{F}}(\boldsymbol{r})$ is the Fisher information density and $\nabla \rho(\boldsymbol{r})$ is the density gradient. Earlier, we have proved that there is an equivalent expression for the Fisher information in terms of the Laplacian of the electron density $\nabla^{2} \rho^{47}$

$$
I_{\mathrm{F}}^{\prime} \equiv \int i_{\mathrm{F}}^{\prime}(\boldsymbol{r}) \mathrm{d} \boldsymbol{r}=-\int \nabla^{2} \rho(\boldsymbol{r}) \ln \rho(\boldsymbol{r}) \mathrm{d} \boldsymbol{r}
$$

Equations (5) and (6) are equal in the sense that they can be derived by partial integration from one to the other, and that the two integrals have the same value. As have been shown, local behaviors of the two integrals, $i_{\mathrm{F}}(\boldsymbol{r})$ and $i_{\mathrm{F}}^{\prime}(\boldsymbol{r})$, are markedly different. More importantly, we have proved the existence of the following rigorous relationship among the three quantities, $s_{\mathrm{S}}(\boldsymbol{r}), i_{\mathrm{F}}(\boldsymbol{r})$, and $i_{\mathrm{F}}^{\prime}(\boldsymbol{r})^{47}$, 


$$
S_{\mathrm{S}}=\int S_{\mathrm{S}}(\boldsymbol{r}) \mathrm{d} \boldsymbol{r}=-N+\frac{1}{4 \pi} \iint \frac{i_{\mathrm{F}}\left(\boldsymbol{r}^{\prime}\right)-i_{\mathrm{F}}^{\prime}\left(\boldsymbol{r}^{\prime}\right)}{\left|\boldsymbol{r}-\boldsymbol{r}^{\prime}\right|} \mathrm{d} \boldsymbol{r} \mathrm{d} \boldsymbol{r}^{\prime}
$$

whose validity has subsequently been verified by numerical results.

The third quantity in the same spirit is the Ghosh-BerkowitzParr (GBP) entropy ${ }^{64}$,

$$
S_{\mathrm{GBP}}=\int \frac{3}{2} k \rho(\boldsymbol{r})\left[c+\ln \frac{t(\boldsymbol{r} ; \rho)}{t_{\mathrm{TF}}(\boldsymbol{r} ; \rho)}\right] \mathrm{d} \boldsymbol{r}
$$

where $t(\boldsymbol{r}, \rho)$ is the kinetic energy density, which is related to the total kinetic energy $T_{\mathrm{S}}$ via

$$
\int t(\boldsymbol{r} ; \rho) \mathrm{d} \boldsymbol{r}=T_{\mathrm{S}}
$$

and $t_{\mathrm{TF}}(\mathbf{r} ; \rho)$ is the Thomas-Fermi kinetic energy density,

$$
t_{\mathrm{TF}}(\boldsymbol{r} ; \rho)=c_{\mathrm{K}} \rho^{5 / 3}(\boldsymbol{r})
$$

with $k$ as the Boltzmann constant (set to be unity for convenience in this work), $c=\frac{5}{3}+\ln \frac{4 \pi c_{\mathrm{K}}}{3}$, and $c_{\mathrm{K}}=\frac{3}{10}\left(3 \pi^{2}\right)^{2 / 3}$. The GBP entropy originates from the effort to transcribe the ground-state density functional theory into a local thermodynamics through the phase-space distribution function $f(\boldsymbol{r}, \boldsymbol{p})$, which is a function of both the electron position $\boldsymbol{r}$ and momentum $\boldsymbol{p}$ as its two basic variables. The conditions of such a recast of DFT into thermodynamics are that the phase-space distribution function is associated with the ground state electron density $\rho(\boldsymbol{r})$ and kinetic energy density $t(\boldsymbol{r} ; \rho)$ through the following relationships

$$
\begin{aligned}
& \rho(\boldsymbol{r})=\int f(\boldsymbol{r}, \boldsymbol{p}) \mathrm{d} \boldsymbol{p} \\
& t(\boldsymbol{r} ; \rho)=\frac{1}{2} \int f(\boldsymbol{r}, \boldsymbol{p}) p^{2} \mathrm{~d} \boldsymbol{p}
\end{aligned}
$$

The specific form of the local kinetic energy $t(r ; \rho)$ used is the following,

$$
t(\boldsymbol{r} ; \rho)=\sum_{i} \frac{1}{8} \frac{\nabla \rho_{i} \cdot \nabla \rho_{i}}{\rho_{i}}-\frac{1}{8} \nabla^{2} \rho
$$

Very recently, three information-theoretic quantities, Rényi entropy, Tsallis entropy, and Onicescu information energy, are introduced as new reactivity descriptors in DFRT ${ }^{65}$. The Rényi entropy of order $n$, where $n \geqslant 0$ and $n \neq 1$, is defined as ${ }^{66}$

$$
R_{n}=\frac{1}{1-n} \ln \left[\int \rho(\boldsymbol{r})^{n} \mathrm{~d} \boldsymbol{r}\right]
$$

When $n$ approaches to 1, the Rényi entropy, Eq.(14), reduces to the Shannon entropy, Eq.(3). The Tsallis entropy of order $n$ is defined as follows, ${ }^{67}$

$$
T_{n}=\frac{1}{n-1}\left[1-\int \rho(\boldsymbol{r})^{n} \mathrm{~d} \boldsymbol{r}\right]
$$

It is a generalization of the standard Boltzmann-Gibbs entropy. The common term in Eqs.(14) and (15) is the integral of the $n$ th power of the electron density, which is called the Onicescu information energy of order $n^{68}$ :

$$
E_{n}=\frac{1}{n-1} \int \rho(\boldsymbol{r})^{n} \mathrm{~d} \boldsymbol{r}
$$

Onicescu introduced this quantity in an attempt to define a finer measure of dispersion distribution than that of Shannon entropy in information theory.

Closely related to the concept of entropy in information theory is the relative entropy, which is a non-symmetric measure of the entropy difference between two probability distribution functions. Well known examples in the literature are the relative Shannon entropy, also called information gain, KullbackLeibler divergence, or information divergence, defined by ${ }^{69}$

$$
S_{r}=\int \rho(\boldsymbol{r}) \ln \frac{\rho(\boldsymbol{r})}{\rho_{0}(\boldsymbol{r})} \mathrm{d} \boldsymbol{r}
$$

and the relative Rényi entropy of order $n^{24}$,

$$
R_{n}^{r}=\frac{1}{n-1} \ln \left[\int \frac{\rho^{n}(\boldsymbol{r})}{\rho_{0}^{n-1}(\boldsymbol{r})} \mathrm{d} \boldsymbol{r}\right]
$$

where $\rho_{0}(\boldsymbol{r})$ is the reference state density satisfying the same normalization condition as $\rho(\boldsymbol{r})$. This reference density can be from the same molecule with different conformation or from the reactant of a chemical reaction when the transition state is investigated.

\subsection{Shape function representation}

Information-theoretic quantities defined in Eqs.(3)-(18) employ the electron density as the probability distribution function. There is another distribution function in DFRT, the shape function $\sigma(\boldsymbol{r})^{1,19,70-72}$, which is related to the electron density $\rho(\boldsymbol{r})$ and the total number of electrons $N$ through the following relationship,

$$
\rho(\boldsymbol{r})=N \sigma(\boldsymbol{r})
$$

with the following normalization condition

$$
\int \sigma(\boldsymbol{r}) \mathrm{d} \boldsymbol{r}=1
$$

Information-theoretic quantities defined in Eqs.(3)-(18) can similarly be redefined with the shape function, yielding ${ }^{19,65}$

$$
\begin{aligned}
& S_{\sigma}=-\int \sigma(\boldsymbol{r}) \ln \sigma(\boldsymbol{r}) \mathrm{d} \boldsymbol{r} \\
& I_{\sigma}=\int \frac{|\nabla \sigma(\boldsymbol{r})|^{2}}{\sigma(\boldsymbol{r})} \mathrm{d} \boldsymbol{r} \\
& I_{\sigma}^{\prime}=-\int\left[\nabla^{2} \sigma(\boldsymbol{r})\right] \ln \sigma(\boldsymbol{r}) \mathrm{d} \boldsymbol{r} \\
& R_{n}^{\sigma}=\frac{1}{1-n} \ln \left[\int \sigma(\boldsymbol{r})^{n} \mathrm{~d} \boldsymbol{r}\right] \\
& T_{n}^{\sigma}=\frac{1}{n-1}\left[1-\int \sigma(\boldsymbol{r})^{n} \mathrm{~d} \boldsymbol{r}\right] \\
& E_{n}^{\sigma}=\frac{1}{n-1} \int \sigma(\boldsymbol{r})^{n} \mathrm{~d} \boldsymbol{r} \\
& S_{r}^{\sigma}=\int \sigma(\boldsymbol{r}) \ln \frac{\sigma(\boldsymbol{r})}{\sigma_{0}(\boldsymbol{r})} \mathrm{d} \boldsymbol{r}
\end{aligned}
$$

and

$$
R_{\sigma, n}^{r}=\frac{1}{n-1} \ln \left[\int \frac{\sigma^{n}(\boldsymbol{r})}{\sigma_{0}^{n-1}(\boldsymbol{r})} \mathrm{d} \boldsymbol{r}\right]
$$

Because of Eq.(19), quantities in these two representations are correlated, except for the GBP entropy, which does not have an 
analytical expression between the two representations. As can be readily proved, we have $\mathrm{e}^{19,65}$

$$
\begin{aligned}
& S_{\sigma}=\frac{S_{S}}{N}+\ln N \\
& I_{\mathrm{F}}=N I_{\sigma} \\
& I_{\mathrm{F}}^{\prime}=N I_{\sigma}^{\prime} \\
& R_{n}-R_{n}^{\sigma}=\frac{n}{1-n} \ln N \\
& T_{n}-N^{n} T_{n}^{\sigma}=\frac{1-N^{n}}{n-1} \\
& E_{n}=N^{n} E_{n}^{\sigma} \\
& S_{r}=N S_{r}^{\sigma}
\end{aligned}
$$

and

$$
R_{n}^{r}-R_{\sigma, n}^{r}=\frac{1}{1-n} \ln N
$$

These rigorous relationships between the two representations of information-theoretic quantities enable us to obtain them interchangeably from one representation to the other.

\subsection{Atoms-in-molecules representation}

Another important aspect of the information-theoretic approach is to reevaluate the above quantities from the perspective of atoms in molecules ${ }^{19,65,73,74}$. To consider atomic contributions of an information-theoretic quantity in a molecular system, three approaches are available to perform atom partitions in molecules. They are Becke's fuzzy atom approach ${ }^{75}$, Bader's zero-flux AIM approach ${ }^{11}$, and Hirshfeld's stockholder approach $^{14}$. The total electron population $N$ of the system is the summation of electron density in each atomic contribution, $N_{\mathrm{A}}$,

$$
N=\sum_{\mathrm{A}} N_{\mathrm{A}}=\sum_{\mathrm{A}} \int_{\Omega_{\mathrm{A}}} \rho_{\mathrm{A}}(\boldsymbol{r}) \mathrm{d} \boldsymbol{r}
$$

and the quantities in Eqs.(3)-(18) can be rewritten as a $^{73,74}$

$$
\begin{aligned}
& S_{\mathrm{S}}=-\sum_{\mathrm{A}} \int_{\Omega_{\mathrm{A}}} \rho_{\mathrm{A}}(\boldsymbol{r}) \ln \rho_{\mathrm{A}}(\boldsymbol{r}) \mathrm{d} \boldsymbol{r} \\
& I_{\mathrm{F}}=\sum_{\mathrm{A}} \int_{\Omega_{\mathrm{A}}} \frac{\left|\nabla \rho_{\mathrm{A}}(\boldsymbol{r})\right|^{2}}{\rho_{\mathrm{A}}(\boldsymbol{r})} \mathrm{d} \boldsymbol{r} \\
& I_{\mathrm{F}}^{\prime}=-\sum_{\mathrm{A}} \int^{2} \rho_{\mathrm{A}}(\boldsymbol{r}) \ln \rho_{\mathrm{A}}(\boldsymbol{r}) \mathrm{d} \boldsymbol{r} \\
& S_{\mathrm{GBP}}=-\sum_{\mathrm{A}} \int_{\Omega_{\mathrm{A}}} \frac{3}{2} k \rho_{\mathrm{A}}(\boldsymbol{r})\left[c+\ln \frac{t\left(\boldsymbol{r} ; \rho_{\mathrm{A}}\right)}{t_{\mathrm{TF}}\left(\boldsymbol{r} ; \rho_{\mathrm{A}}\right)}\right] \mathrm{d} \boldsymbol{r} \\
& E_{n}=\frac{1}{n-1} \sum_{\mathrm{A}} \int_{\Omega_{\mathrm{A}}} \rho_{\mathrm{A}}^{n}(\boldsymbol{r}) \mathrm{d} \boldsymbol{r}
\end{aligned}
$$

and

$$
S_{r}=\sum_{\mathrm{A}} \int_{\Omega_{\mathrm{A}}} \rho_{\mathrm{A}}(\boldsymbol{r}) \frac{\rho_{\mathrm{A}}(\boldsymbol{r})}{\rho_{\mathrm{A}}^{0}(\boldsymbol{r})} \mathrm{d} \boldsymbol{r}
$$

where $\rho_{\mathrm{A}}$ is the electron density on atom (or group) A in a molecule, whose total molecular electron density is $\rho(\boldsymbol{r}), \rho_{\mathrm{A}}^{0}$ is the counterpart of atom (or group) $\mathrm{A}$ in the reference state, which can be neutral atom, or ion, or group, etc., and $\Omega_{\mathrm{A}}$ is the atomic basin of atom A in the molecule. The counterpart in terms of the shape function can be derived similarly.

All these formulas shown above in the information-theoretic approach as well as the three partition schemes for atoms in molecules have been successfully implemented in the Multiwfn package $^{76}$, whose reliability and applicability have extensively been tested and confirmed.

All the quantities introduced above from the informationtheoretic approach share the same characteristics: they are all simple density functionals! Different from the density functionals for energy components such as the noninteracting kinetic energy $T_{\mathrm{S}}$ and the exchange-correlation energy $E_{\mathrm{xc}}$, where many different levels of approximations have been introduced ${ }^{1}$, the form of these density functionals is not only analytical but also much simpler. Still, they all have their sound origin from information theory. Do they provide any insight from the perspective of chemical reactivity? Also, except the known relationship in Eq.(7), are they correlated in any manner?

We will address these and other questions in the following sections.

\section{Principle of extreme physical information}

The Euler equation of DFT for a real interacting system $\mu=\delta E / \delta \rho$ can be recast in the model, non-interacting system and becomes ${ }^{1}$

$$
\mu=\frac{\delta T_{\mathrm{S}}}{\delta \rho(\boldsymbol{r})}+v_{\mathrm{KS}}(\boldsymbol{r})
$$

where $T_{\mathrm{S}}, v_{\mathrm{KS}}$, and $\mu$ are the noninteracting kinetic energy, Kohn-Sham potential, and chemical potential arising from the constraint that the total number of electrons must be fixed to $N$, Eq.(4). Furthermore, the noninteracting kinetic energy can be divided into two pieces,

$$
T_{\mathrm{S}}=T_{\mathrm{W}}+T_{\mathrm{P}}
$$

where $T_{\mathrm{W}}$ is the Weizsäcker kinetic energy ${ }^{77}$

$$
T_{\mathrm{W}}=\frac{1}{8} \int \frac{|\nabla \rho(\boldsymbol{r})|^{2}}{\rho(\boldsymbol{r})} \mathrm{d} \boldsymbol{r}
$$

and $T_{\mathrm{P}}$ is the Pauli energy due to the Pauli Exclusion Principle ${ }^{78,79}$. With Eq.(45), the Euler equation becomes

$$
\mu=\frac{\delta T_{\mathrm{W}}}{\delta \rho(\boldsymbol{r})}+v_{\mathrm{P}}(\boldsymbol{r})+v_{\mathrm{KS}}(\boldsymbol{r})
$$

where $v_{\mathrm{P}}$ is the Pauli potential defined as the functional of derivative of $T_{\mathrm{P}}$ with respect to the electron density. With the help that

$$
\begin{aligned}
\frac{\delta T_{\mathrm{W}}}{\delta \rho(\boldsymbol{r})} & =\frac{1}{8}\left(\frac{\nabla \rho(\boldsymbol{r})}{\rho(\boldsymbol{r})}\right)^{2}-\frac{1}{4} \frac{\nabla^{2} \rho(\boldsymbol{r})}{\rho(\boldsymbol{r})} \\
& =\rho^{-1 / 2}(\boldsymbol{r})\left(-\frac{1}{2} \nabla^{2}\right) \rho^{-1 / 2}(\boldsymbol{r})
\end{aligned}
$$

Eq.(47) reads

$$
\left(-\frac{1}{2} \nabla^{2}+v_{\mathrm{P}}(\boldsymbol{r})+v_{\mathrm{KS}}(\boldsymbol{r})\right) \rho^{1 / 2}(\boldsymbol{r})=\mu \rho^{1 / 2}(\boldsymbol{r})
$$

Equation (49) is similar to the Kohn-Sham equation, but it does 
not require the introduction of any orbitals. It is the working formula for the orbital-free DFT (OF DFT) approach in $\mathrm{DFT}^{2-4}$.

This OF-DFT equation can, however, be derived from the principle of extreme physical information, which states that the "physical information" $\mathrm{K}$ of a system should be extreme: ${ }^{26}$

$$
K=I_{\mathrm{F}}-J=\text { extreme }
$$

where $I_{\mathrm{F}}$ is the Fisher information, $J$ is the set of constraints that are to be imposed, and $K$ is the difference between $I_{\mathrm{F}}$ and $J$, called the "physical information". According to the literature ${ }^{80,81}$, these constraints are three-fold. (a) The total wave function of the system is antisymmetric. As shown by the Nagy and others ${ }^{80,81}$, this condition generates the Pauli potential $v_{\mathrm{P}}(\boldsymbol{r})$. (b) The total density of the noninteracting system should be the same as that of the interacting system. Same as the Kohn-Sham equation, this constraints yields the Kohn-Sham potential $v_{\mathrm{KS}}(\boldsymbol{r})$. (c) Lastly, the total electron density is normalized to $N$, Eq.(4), which results in a Lagrange multiplier $\mu$, the chemical potential of the system.

Put together, that is to say, minimizing the Fisher information subject to the above three constraints should give us the same Euler equation in Eq.(49) as what we have obtained from the OF-DFT approach. What the principle of extreme physical information tells us is that one does not have to use the electronic energy when deriving Euler equation in DFT. It could be substituted by information. In other words, information alone is adequate in understanding all properties of the system.

\section{Scaling properties of information- theoretic quantities}

Quantities from the information-theoretic approach are simple density functionals. One of the first properties of these functionals one wishes to examine is their scaling properties ${ }^{82-84}$. Scaling properties of energy density functionals in DFT have been extensively studied in the literature ${ }^{85,86}$. There are two distinct categories of the scaling, one via scaling the coordinate variable and the other through scaling the electron density. One can also scale both coordinate and density variables at the same time, leading to the so-called hybrid scaling ${ }^{82-86}$.

Homogeneity of a functional $Q[\rho]$ of degree $m$ in (or "with respect to") coordinate scaling is defined as

$$
Q\left[\rho_{\gamma}\right]=\gamma^{m} Q[\rho]
$$

where

$$
\rho_{\gamma}(\boldsymbol{r})=\gamma^{3} \rho(\gamma \boldsymbol{r})
$$

Equation (52) is imposed as the condition of the coordinate scaling because it will always keep the scaled density $\rho(\gamma \boldsymbol{r})$ normalized to $N$. It has been previously proved that for a wellbehaved functional, Eq.(51) is equivalent to

$$
-\int \rho(\boldsymbol{r}) \boldsymbol{r} \cdot \nabla\left(\frac{\delta Q[\rho]}{\delta \rho(\boldsymbol{r})}\right) \mathrm{d} \boldsymbol{r}=m Q[\rho]
$$

Examples of homogeneous functionals in coordinate scaling include the kinetic and exchange energy functionals. There are numerous studies in the literature to express the correlation en- ergy functionals in terms of homogeneous functionals in coordinate scaling.

A functional $Q[\rho]$ is homogeneous of degree $n$ in density scaling if it satisfies the following condition:

$$
Q[\zeta \rho]=\zeta^{n} Q[\rho] \text {. }
$$

Another definition of the homogeneity with respect to density scaling is as follows,

$$
\int \rho(\boldsymbol{r}) \frac{\delta Q[\rho]}{\delta \rho(\boldsymbol{r})} \mathrm{d} \boldsymbol{r}=n Q[\rho]
$$

Examples of homogeneous functionals in density scaling include the Thomas-Fermi formula for the kinetic energy and the Dirac formula for the exchange energy. The density scaling is related to a quantity's extensive/intensive nature. For example, Fisher information is homogeneous of degree one in density scaling because if one scales the density by $\zeta$ times, $I_{\mathrm{F}}$ is amplified by $\zeta$ times as well, that is, $I_{\mathrm{F}}[\zeta \rho]=\zeta I_{\mathrm{F}}[\rho]$. However, for the Shannon entropy, no such relationship can be obtained when the density is scaled by $\zeta$ times. Instead, we get

$$
S_{\mathrm{S}}[\zeta \rho]=-N \zeta \ln \zeta+\zeta S_{\mathrm{S}}[\rho]
$$

Therefore, Shannon entropy is not a homogeneous functional in density scaling. This is also true for the GBP entropy functional, which is not known to satisfy any homogeneity relations in both density and coordinate scaling. For the alternative definition of the Fisher information defined in Eq.(6), we have the similar density scaling property as the Shannon entropy. So, even though the two forms of Fisher information yield the same molecular value, not only their local behaviors are vastly different, but their scaling properties are also well. Similarly, we can prove that Rényi entropy and Tsallis entropy are not homogeneous functionals in density scaling, but the Onicescu information energy of order $n$ is homogeneous of degree $n$ in density scaling. For counterpart quantities in the shape function representation, similar conclusions can be obtained.

While these analyses of scaling properties are helpful in understanding analytical properties of these quantities, it is not straightforward to verify their validity with numerical results. To overcome this problem, in our recent work ${ }^{19}$, we proposed to approach it in a little different manner. Instead of scaling the electron density for the same molecule, we consider the scaling property of these quantities in different molecular systems with respect to the total electron population. At the meanwhile, we also examine the scaling property at the atoms-in-molecules level using three different ways of atomic partition scheme from the literature, Becke's fuzzy atom approach, Bader's zeroflux AIM approach, and Hirshfeld's stockholder approach. As an example, shown in Table 1 are the numerical values for a few quantities for the first and second row neutral atoms, with the correlation coefficient with respect to the total number of electrons shown in the last row. As can be seen from Table 1, linear dependences of $I_{\sigma}, I_{\sigma}^{\prime}, S_{\mathrm{GBP}}$, and $S_{\mathrm{GBP}}^{\prime}$ are remarkable, indicating that there exist strong scaling properties for these quantities $^{19}$. Table 2 displays the atomic values for Rényi entropy, Tsallis entropy, Onicescu information energy of orders 2 
Table 1 Information-theoretic quantities (in atomic units) for first and second-row neutral atoms

\begin{tabular}{|c|c|c|c|c|c|c|c|c|c|}
\hline Atom & $N$ & $S_{\mathrm{S}}$ & $S_{\sigma}$ & $I_{\mathrm{F}}$ & $I_{\sigma}$ & $I_{\mathrm{F}}^{\prime}$ & $I_{\sigma}^{\prime}$ & $S_{\mathrm{GBP}}$ & $S_{\mathrm{GBP}}^{\prime}$ \\
\hline $\mathrm{H}$ & 1 & 4.21 & 4.21 & 3.97 & 3.97 & 3.97 & 3.97 & 7.80 & 7.41 \\
\hline $\mathrm{He}$ & 2 & 4.10 & 2.74 & 22.90 & 11.45 & 22.90 & 11.45 & 14.42 & 13.42 \\
\hline $\mathrm{Li}$ & 3 & 7.69 & 3.66 & 57.39 & 19.13 & 57.36 & 19.12 & 22.19 & 21.06 \\
\hline $\mathrm{Be}$ & 4 & 8.85 & 3.6 & 109.10 & 27.28 & 109.10 & 27.27 & 28.82 & 27.49 \\
\hline B & 5 & 8.83 & 3.38 & 175.48 & 35.10 & 175.47 & 35.09 & 36.07 & 34.34 \\
\hline $\mathrm{C}$ & 6 & 7.85 & 3.1 & 255.96 & 42.66 & 255.96 & 42.66 & 43.08 & 40.91 \\
\hline $\mathrm{N}$ & 7 & 6.14 & 2.82 & 349.61 & 49.94 & 349.61 & 49.94 & 49.95 & 47.30 \\
\hline $\mathrm{O}$ & 8 & 3.91 & 2.57 & 460.35 & 57.54 & 460.35 & 57.54 & 56.37 & 53.35 \\
\hline $\mathrm{F}$ & 9 & 1.11 & 2.32 & 585.14 & 65.02 & 585.14 & 65.02 & 62.72 & 59.29 \\
\hline $\mathrm{Ne}$ & 10 & -2.18 & 2.08 & 723.32 & 72.33 & 723.32 & 72.33 & 69.02 & 65.15 \\
\hline $\mathrm{Na}$ & 11 & -0.88 & 2.32 & 881.74 & 80.16 & 881.70 & 80.15 & 76.85 & 72.54 \\
\hline $\mathrm{Mg}$ & 12 & -1.25 & 2.38 & 1058.09 & 88.17 & 1058.07 & 88.17 & 83.64 & 78.92 \\
\hline $\mathrm{Al}$ & 13 & -1.84 & 2.42 & 1251.46 & 96.27 & 1251.44 & 96.26 & 90.56 & 85.86 \\
\hline $\mathrm{Si}$ & 14 & -3.26 & 2.41 & 1460.74 & 104.34 & 1460.74 & 104.34 & 97.33 & 92.50 \\
\hline $\mathrm{P}$ & 15 & -5.22 & 2.36 & 1686.21 & 112.41 & 1686.21 & 112.41 & 104.01 & 98.94 \\
\hline $\mathrm{S}$ & 16 & -7.61 & 2.30 & 1928.83 & 120.55 & 1928.83 & 120.55 & 110.22 & 105.02 \\
\hline $\mathrm{Cl}$ & 17 & -10.36 & 2.22 & 2187.77 & 128.69 & 2187.77 & 128.69 & 116.41 & 111.01 \\
\hline $\mathrm{Ar}$ & 18 & -13.53 & 2.14 & 2463.43 & 136.86 & 2463.43 & 136.86 & 122.47 & 116.90 \\
\hline$R^{2}$ & & 0.8265 & 0.6660 & 0.9376 & 0.9998 & 0.9376 & 0.9998 & 0.9997 & 0.9997 \\
\hline
\end{tabular}

and 3, respectively, with $R^{2}$ values with respect to the total atomic number shown at the end as well. Because they are not linear functionals with respect to the total number of electrons, their $R^{2}$ values are not close to unit ${ }^{65}$.

Table 3 exhibits the numerical results for a total of 42 neutral molecules, with the correlation coefficients also shown at the end. ${ }^{19}$ Although the $R^{2}$ values are not as good as the atomic case for $I_{\sigma}$ and $I_{\sigma}^{\prime}$, they are still pretty decent. Plus, for $S_{\mathrm{GBP}}$, and $S_{\mathrm{GBP}}^{\prime}$, same as the atomic case, the linear correlations are still extremely strong.

At the atoms-in-molecule level of theory, the three atom partition schemes yield similar, though not identical, results, as shown in Table 4 by their correlation coefficients with different information-theoretic quantities ${ }^{19}$. Fig. 1 demonstrates three of these strong correlations for carbon and hydrogen atoms in the 42 systems studied, using Becke's fuzzy atom partitioning approach as an example. We also studied the behavior of these quantities as a function of molecular conformation change at both molecular and atoms-in-molecules levels. Shown in Fig.2, as an example, is the profiles of the second-order Onicescu information energy as a function of the $\angle \mathrm{HCCH}$ dihedral angle for the ethane molecule, which is change from $0^{\circ}$ (staggered isomer) to $360^{\circ}$ at both levels ${ }^{65}$.

These scaling properties with respect to the total number of electron populations at both the molecular and atoms in molecules levels have also been investigated and confirmed in a few reaction systems ${ }^{20-22}$.

\section{Quantification of steric effect}

Steric effect is one of the most widely used concepts in chemistry. It originates from the fact that each atom in a mo- lecule occupies a certain amount of space. When atoms are brought together, hindrance will be induced in the expense of shape, energy, reactivity, etc. According to Weisskopf ${ }^{87}$, steric effect is resulted from the "kinetic energy pressure" in atoms and molecules. Description of the effect employing Pauli exchange interactions from the wave function theory is available ${ }^{88}$, but a satisfactory quantification is still lacking. Earlier, we proposed to quantify the steric effect within the framework of density functional reactivity theory with the Weizsäcker kinetic energy $T_{\mathrm{W}}{ }^{15}$,

$$
E_{\mathrm{S}}=T_{\mathrm{W}} \equiv \frac{1}{8} \int \frac{|\nabla \rho(\boldsymbol{r})|^{2}}{\rho(\boldsymbol{r})} \mathrm{d} \boldsymbol{r}
$$

which is related to the Fisher information by a factor of 8 ,

$$
I_{\mathrm{F}} \equiv \int \frac{|\nabla \rho(\boldsymbol{r})|^{2}}{\rho(\boldsymbol{r})} \mathrm{d} \boldsymbol{r}=8 T_{\mathrm{W}}
$$

This approach of quantifying the steric effect was based on the assumption that the total electronic energy of a system comes from the independent contributions of three physiochemical effects, steric, electrostatic, and quantum due to the exchangecorrelation effect ${ }^{15}$ :

$$
E \equiv E_{\mathrm{s}}+E_{\mathrm{e}}+E_{\mathrm{q}}
$$

If the above assumption is valid, we can prove that using the Weizsäcker kinetic energy (i.e., also the Fisher information) to express the steric effect, Eq.(57), should be true.

The proof is straightforward, shown below. Since in DFT, we know that ${ }^{1}$

$$
E=T_{\mathrm{S}}+V_{\mathrm{ne}}+J+E_{\mathrm{xc}}+V_{\mathrm{nn}}
$$

where $T_{\mathrm{S}}, V_{\mathrm{ne}}, J, V_{\mathrm{nn}}$, and $E_{\mathrm{xc}}$ stand for the noninteracting kinetic, nuclear-electron attraction, classical electron-electron Cou- 
Table 2 Atomic values of new information-theoretic quantities (in atomic unit) obtained with the total electron density

\begin{tabular}{|c|c|c|c|c|c|c|c|}
\hline Atom & $N$ & $E_{2}$ & $E_{3}$ & $R_{2}$ & $R_{3}$ & $T_{2}$ & $T_{3}$ \\
\hline $\mathrm{H}$ & 1 & 0.039 & 0.002 & 1.41 & 1.21 & 0.96 & 0.50 \\
\hline $\mathrm{He}$ & 2 & 0.763 & 0.389 & 0.12 & 0.05 & 0.24 & 0.11 \\
\hline $\mathrm{Li}$ & 3 & 3.139 & 6.170 & -0.50 & -0.55 & -2.14 & -5.67 \\
\hline $\mathrm{Be}$ & 4 & 8.40 & 42.76 & -0.92 & -0.97 & -7.40 & -42.26 \\
\hline $\mathrm{C}$ & 6 & 31.87 & 580.9 & -1.50 & -1.53 & -30.87 & -580.37 \\
\hline $\mathrm{N}$ & 7 & 52.69 & 1538.8 & -1.72 & -1.74 & -51.69 & -1538.28 \\
\hline $\mathrm{O}$ & 8 & 81.65 & 3567.3 & -1.91 & -1.93 & -80.65 & -3566.82 \\
\hline $\mathrm{F}$ & 9 & 120.3 & 7455.5 & -2.08 & -2.09 & -119.3 & -7455.0 \\
\hline $\mathrm{Ne}$ & 10 & 170.5 & 14377.0 & -2.23 & -2.23 & -169.5 & -14376.5 \\
\hline $\mathrm{Na}$ & 11 & 234.0 & 26194.0 & -2.37 & -2.36 & -233.0 & -26193.5 \\
\hline $\mathrm{Mg}$ & 12 & 313.0 & 45493.0 & -2.50 & -2.48 & -312.0 & -45492.5 \\
\hline $\mathrm{Si}$ & 14 & 523.3 & 120384.6 & -2.72 & -2.69 & -522.3 & -120384.1 \\
\hline $\mathrm{P}$ & 15 & 658.1 & 185814.7 & -2.82 & -2.79 & -657.1 & -185814.2 \\
\hline $\mathrm{S}$ & 16 & 815.3 & 278831.0 & -2.91 & -2.87 & -814.3 & -278830.5 \\
\hline $\mathrm{Cl}$ & 17 & 996.0 & 407976.6 & -3.00 & -2.96 & -995.0 & -407976.1 \\
\hline $\mathrm{Ar}$ & 18 & 1203.0 & 583504.5 & -3.08 & -3.03 & -1202.0 & -583504.0 \\
\hline $\mathrm{K}$ & 19 & 1440.9 & 820114.8 & -3.16 & -3.11 & -1439.9 & -820114.3 \\
\hline $\mathrm{Ca}$ & 20 & 1708.0 & 1132761 & -3.23 & -3.18 & -1707.0 & -1132761 \\
\hline $\mathrm{Cr}$ & 24 & 3094.7 & 3531757 & -3.49 & -3.42 & -3093.7 & -3531757 \\
\hline $\mathrm{Mn}$ & 25 & 3530.3 & 4544622 & -3.55 & -3.48 & -3529.3 & -4544622 \\
\hline $\mathrm{Co}$ & 27 & 4533.3 & 7324860 & -3.66 & -3.58 & -4532.3 & -7324860 \\
\hline $\mathrm{Ni}$ & 28 & 5105.7 & 9187318 & -3.71 & -3.63 & -5104.7 & -9187318 \\
\hline $\mathrm{Zn}$ & 30 & 6386.7 & 14073009 & -3.81 & -3.72 & -6385.7 & -14073009 \\
\hline $\mathrm{Ge}$ & 32 & 7889.8 & 20976835 & -3.90 & -3.81 & -7888.8 & -20976835 \\
\hline As & 33 & 8725.5 & 25385817 & -3.94 & -3.85 & -8724.5 & -25385816 \\
\hline $\mathrm{Se}$ & 34 & 9621.0 & 30546397 & -3.98 & -3.89 & -9620.0 & -30546397 \\
\hline $\mathrm{Br}$ & 35 & 10577.7 & 36545294 & -4.02 & -3.93 & -10576.7 & -36545293 \\
\hline $\mathrm{Kr}$ & 36 & 11603.3 & 43529405 & -4.06 & -3.97 & -11602.3 & -43529404 \\
\hline$R^{2}$ & & 0.834 & 0.653 & 0.806 & 0.806 & 0.834 & 0.653 \\
\hline
\end{tabular}

lomb repulsion, nuclear-nuclear repulsion, and exchange-correlation energy density functionals, respectively. Three components, $V_{\text {ne }}, V_{\mathrm{nn}}$, and $J$, are of the electrostatic nature, $E_{\mathrm{e}}=V_{\mathrm{ne}}+J+$ $V_{\mathrm{nn}}$. For the quantum part, we have

$$
E_{\mathrm{q}}=E_{\mathrm{xc}}+E_{\text {Pauli }}=E_{\mathrm{xc}}+T_{\mathrm{S}}-T_{\mathrm{W}}
$$

where the Pauli energy is ${ }^{78,79}$,

$$
E_{\text {Pauli }} \equiv T_{\mathrm{S}}-T_{\mathrm{W}}
$$

which denotes the portion of the kinetic energy that embodies the effect from the antisymmetric requirement of the total wave function by the Pauli Exclusion Principle. Put together, we have the density-based formulation of the steric energy ${ }^{15}$,

$$
E_{\mathrm{S}}=E-E_{\mathrm{e}}-E_{\mathrm{q}}=T_{\mathrm{W}} \equiv \frac{1}{8} \int \frac{|\nabla \rho(\boldsymbol{r})|^{2}}{\rho(\boldsymbol{r})} \mathrm{d} \boldsymbol{r}=8 I_{\mathrm{F}}
$$

So, the validity of Eq.(57) solely depends on the assumption of Eq.(59). If Eq.(59) is true, so is Eq.(57).

The physical meaning of the above quantification of the steric effect is that it represents a hypothetical state where electrons are assumed to be bosons and $E_{\mathrm{s}}$ is simply the total energy of this state excluding contributions from other two ef- fects, electrostatic and quantum. If the density of the hypothetical boson state were the same as that of the fermionic state, $\rho(\boldsymbol{r})$, the total wave function of the hypothetical state would just be $\sqrt{(\rho(r) / N)}$, where $N$ is the number of electrons. The total kinetic energy of the hypothetical state, from which Weisskopf's "kinetic energy pressure" ${ }^{\text {87 }}$ for the steric effect is calculated, is simply Eq.(57).

From the three-dimensional space point of view, in the above hypothetical state, when all electrons in the ground state are squeezed into the lowest orbital, the space occupied by electrons should be minimal. Still, different atoms with different electrons would occupy different amount of space. Henceforth, the space withheld by the hypothetical state and represented by $E_{\mathrm{s}}$ should be an intrinsic property of the system ${ }^{15}$.

From the Fisher information point of view, which is a measure of the localization of the electron density distribution, this new definition of the steric effect, Eq.(57), quantifies spikes of electron densities around atomic nuclei. According to the principle of extreme physical information discussed above, the Fisher information should be minimized subject to the validity 
Table 3 Information-theoretic quantities (in atomic units) for $\mathbf{4 2}$ molecular systems

\begin{tabular}{|c|c|c|c|c|c|c|c|c|c|}
\hline Molecule & $N$ & $S_{\mathrm{S}}$ & $S_{\sigma}$ & $I_{\mathrm{F}}$ & $I_{\sigma}$ & $I_{\mathrm{F}}^{\prime}$ & $I_{\sigma}^{\prime}$ & $S_{\mathrm{GBP}}$ & $I_{\mathrm{GBP}}^{\prime}$ \\
\hline $\mathrm{CH}_{2}=\mathrm{CH}_{2}$ & 16 & 21.86 & 4.14 & 507.98 & 31.75 & 507.98 & 31.75 & 109.16 & 104.74 \\
\hline $\mathrm{FHC}=\mathrm{CH}_{2}$ & 24 & 18.50 & 3.95 & 1082.22 & 45.09 & 1082.22 & 45.09 & 164.23 & 156.66 \\
\hline $\mathrm{MeHC}=\mathrm{CH}_{2}$ & 24 & 32.02 & 4.51 & 759.74 & 31.66 & 759.74 & 31.66 & 163.16 & 156.77 \\
\hline $\mathrm{CH}_{2}=\mathrm{CH}(\mathrm{CN})$ & 28 & 27.95 & 4.33 & 1092.74 & 39.03 & 1092.74 & 39.03 & 191.74 & 183.11 \\
\hline $\mathrm{CH}_{2}=\mathrm{CH}(\mathrm{CHO})$ & 30 & 28.48 & 4.35 & 1205.85 & 40.19 & 1205.85 & 40.19 & 204.96 & 195.95 \\
\hline butadiene & 30 & 37.09 & 4.64 & 1005.62 & 33.52 & 1005.62 & 33.52 & 204.22 & 195.92 \\
\hline $\mathrm{F}_{2} \mathrm{C}=\mathrm{CH}_{2}$ & 32 & 15.08 & 3.94 & 1655.77 & 51.74 & 1655.77 & 51.74 & 219.24 & 208.49 \\
\hline $\mathrm{FHC}=\mathrm{CHF}$ & 32 & 15.15 & 3.94 & 1656.65 & 51.77 & 1656.65 & 51.77 & 219.29 & 208.62 \\
\hline trans $-\mathrm{MeHC}=\mathrm{CHMe}$ & 32 & 42.16 & 4.78 & 1011.54 & 31.61 & 1011.54 & 31.61 & 217.16 & 208.81 \\
\hline cis-MeHC $=\mathrm{CHMe}$ & 32 & 42.12 & 4.78 & 1011.32 & 31.60 & 1011.32 & 31.60 & 217.12 & 208.77 \\
\hline $\mathrm{Me}_{2} \mathrm{C}=\mathrm{CH}_{2}$ & 32 & 42.14 & 4.78 & 1011.33 & 31.60 & 1011.33 & 31.6 & 217.11 & 208.74 \\
\hline$\left(\mathrm{CH}_{3} \mathrm{O}\right) \mathrm{CH}=\mathrm{CH}_{2}$ & 32 & 33.64 & 4.52 & 1210.4 & 37.83 & 1210.4 & 37.83 & 217.99 & 208.83 \\
\hline cyclopentadiene & 36 & 40.99 & 4.72 & 1247.88 & 34.66 & 1247.88 & 34.66 & 244.39 & 234.59 \\
\hline pyrrole & 36 & 36.63 & 4.60 & 1336.36 & 37.12 & 1336.36 & 37.12 & 244.73 & 234.45 \\
\hline 2-methyl-butadiene & 38 & 47.15 & 4.88 & 1257.07 & 33.08 & 1257.07 & 33.08 & 258.12 & 247.86 \\
\hline 1-methyl-butadiene & 38 & 47.22 & 4.88 & 1257.29 & 33.09 & 1257.29 & 33.09 & 258.19 & 247.93 \\
\hline $\mathrm{CH}_{2} \mathrm{CH}=\mathrm{CHOBH}_{3}$ & 38 & 43.07 & 4.77 & 1383.89 & 36.42 & 1383.89 & 36.42 & 259.20 & 248.28 \\
\hline $\mathrm{CH}_{2}=\mathrm{CH}\left(\mathrm{NO}_{2}\right)$ & 38 & 26.23 & 4.33 & 1747.77 & 45.99 & 1747.77 & 45.99 & 259.88 & 247.91 \\
\hline $\mathrm{F}_{2} \mathrm{C}=\mathrm{CHF}$ & 40 & 11.76 & 3.98 & 2230.30 & 55.76 & 2230.29 & 55.76 & 274.32 & 260.48 \\
\hline $\mathrm{Me}_{2} \mathrm{C}=\mathrm{CHMe}$ & 40 & 52.23 & 4.99 & 1262.88 & 31.57 & 1262.88 & 31.57 & 271.06 & 260.73 \\
\hline$(\mathrm{Me})_{2} \mathrm{NCH}=\mathrm{CH}_{2}$ & 40 & 48.16 & 4.89 & 1352.86 & 33.82 & 1352.86 & 33.82 & 271.44 & 260.74 \\
\hline $\mathrm{EtOCH}=\mathrm{CH}_{2}$ & 40 & 43.84 & 4.78 & 1462.27 & 36.56 & 1462.27 & 36.56 & 271.98 & 260.84 \\
\hline$(\mathrm{CN})_{2} \mathrm{C}=\mathrm{CH}_{2}$ & 40 & 34.10 & 4.54 & 1677.67 & 41.94 & 1677.67 & 41.94 & 274.35 & 261.51 \\
\hline benzene & 42 & 45.62 & 4.82 & 1493.23 & 35.55 & 1493.22 & 35.55 & 285.21 & 273.59 \\
\hline $\mathrm{CH}_{3} \mathrm{COOCH}=\mathrm{CH}_{2}$ & 46 & 40.02 & 4.70 & 1907.55 & 41.47 & 1907.55 & 41.47 & 313.61 & 299.85 \\
\hline $\mathrm{CH}_{2}=\mathrm{C}\left(\mathrm{NO}_{2}\right)\left(\mathrm{NH}_{2}\right)$ & 46 & 32.03 & 4.53 & 2088.19 & 45.40 & 2088.19 & 45.40 & 314.23 & 299.77 \\
\hline $\mathrm{F}_{2} \mathrm{C}=\mathrm{CF}_{2}$ & 48 & 8.390 & 4.05 & 2803.95 & 58.42 & 2803.94 & 58.42 & 329.35 & 312.36 \\
\hline $\mathrm{Me}_{2} \mathrm{C}=\mathrm{CMe}_{2}$ & 48 & 62.26 & 5.17 & 1514.31 & 31.55 & 1514.31 & 31.55 & 324.94 & 312.67 \\
\hline maleic anhydride & 50 & 30.29 & 4.52 & 2344.33 & 46.89 & 2344.33 & 46.89 & 341.72 & 325.65 \\
\hline $\mathrm{C}_{6} \mathrm{H}_{5}-\mathrm{Me}$ & 50 & 55.75 & 5.03 & 1744.84 & 34.90 & 1744.84 & 34.90 & 339.17 & 325.58 \\
\hline $\mathrm{C}_{6} \mathrm{H}_{5}-\mathrm{F}$ & 50 & 42.24 & 4.76 & 2067.27 & 41.35 & 2067.27 & 41.35 & 340.28 & 325.50 \\
\hline $\mathrm{C}_{6} \mathrm{H}_{5}-\mathrm{NH}_{2}$ & 50 & 51.52 & 4.94 & 1834.36 & 36.69 & 1834.36 & 36.69 & 339.63 & 325.53 \\
\hline $\mathrm{C}_{6} \mathrm{H}_{5}-\mathrm{OH}$ & 50 & 47.05 & 4.85 & 1942.83 & 38.86 & 1942.83 & 38.86 & 340.04 & 325.54 \\
\hline $\mathrm{C}_{6} \mathrm{H}_{5}-\mathrm{CN}$ & 54 & 51.70 & 4.95 & 2077.92 & 38.48 & 2077.92 & 38.48 & 367.78 & 351.94 \\
\hline $\mathrm{C}_{6} \mathrm{H}_{5}-\mathrm{HCO}$ & 56 & 52.18 & 4.96 & 2190.81 & 39.12 & 2190.81 & 39.12 & 380.96 & 364.77 \\
\hline$(\mathrm{EtO})_{2} \mathrm{C}=\mathrm{CH}_{2}$ & 64 & 65.59 & 5.18 & 2415.68 & 37.75 & 2415.68 & 37.74 & 434.62 & 416.76 \\
\hline$(\mathrm{CN})_{2} \mathrm{C}=\mathrm{C}(\mathrm{CN})_{2}$ & 64 & 46.42 & 4.88 & 2847.48 & 44.49 & 2847.48 & 44.49 & 439.60 & 418.30 \\
\hline $\mathrm{C}_{6} \mathrm{H}_{5}-\mathrm{NO}_{2}$ & 64 & 49.90 & 4.94 & 2732.57 & 42.70 & 2732.57 & 42.70 & 435.86 & 416.70 \\
\hline $\mathrm{C}_{6} \mathrm{H}_{5}-\mathrm{CF}_{3}$ & 74 & 45.74 & 4.92 & 3468.00 & 46.86 & 3468.00 & 46.86 & 504.22 & 481.17 \\
\hline $\mathrm{C}_{6} \mathrm{H}_{5}-\mathrm{N}(\mathrm{Me})_{3}$ & 74 & 80.98 & 5.40 & 2594.49 & 35.06 & 2594.49 & 35.06 & 500.69 & 481.51 \\
\hline 2,3-diacetoxy-1,3-butadiene & 90 & 73.28 & 5.31 & 3804.01 & 42.27 & 3804.01 & 42.27 & 612.98 & 586.03 \\
\hline$\left(\mathrm{C}_{2} \mathrm{H}_{5} \mathrm{COO}\right)_{2} \mathrm{C}=\mathrm{CH}_{2}$ & 92 & 78.58 & 5.38 & 3810.73 & 41.42 & 3810.73 & 41.42 & 626.05 & 598.96 \\
\hline$R^{2}$ & & 0.5118 & 0.4744 & 0.8722 & 0.0425 & 0.8722 & 0.0425 & 0.9999 & 1.0000 \\
\hline
\end{tabular}

of the three constraints ${ }^{26,80,81}$. This means that when a molecular system is formed, atoms in molecules tend to occupy the minimal amount of space, if the three conditions mentioned in Section 4 are satisfied.

There are a few nice properties associated with this quantification of the steric effect in DFRT. ${ }^{15}$ For instance, the integrand of Eq.(57) is non-negative everywhere, and thus repulsive in nature. It vanishes for the case of a homogeneous electron gas. It is extensive because it is homogeneous of degree one in density scaling, as shown in the above section, so the larger the system, the larger the steric repulsion. If Bader's atoms-in-molecules approach or any of other atom partition methods is ad- 
Table 4 Correlation coefficient values $\left(R^{2}\right)$ of all least square fitts with respect to the total number of electron populations for the informationtheoretic quantities presented in this work at atoms-in-molecules level with AIM, Becke, and Hirshfeld partitionings

\begin{tabular}{|c|c|c|c|c|c|c|}
\hline & \multicolumn{3}{|c|}{$\mathrm{C}$} & \multicolumn{3}{|c|}{$\mathrm{H}$} \\
\hline & AIM & Becke & Hirshfeld & AIM & Becke & Hirshfeld \\
\hline$S_{\mathrm{S}}$ & 0.9590 & 0.9255 & 0.7195 & 0.9938 & 0.9686 & 0.8905 \\
\hline$S_{\sigma}$ & 0.9700 & 0.9290 & 0.7501 & 0.9541 & 0.8956 & 0.6520 \\
\hline$I_{\mathrm{F}}$ & 0.6349 & 0.0069 & 0.5690 & 0.8385 & 0.3022 & 0.2982 \\
\hline$I_{\sigma}$ & 0.9932 & 0.9885 & 0.9714 & 0.8869 & 0.7848 & 0.8011 \\
\hline$I_{\mathrm{F}}^{\prime}$ & 0.6361 & 0.1273 & 0.7051 & 0.8394 & 0.0008 & 0.5454 \\
\hline$I_{\sigma}^{\prime}$ & 0.9932 & 0.9746 & 0.9397 & 0.8877 & 0.2328 & 0.6954 \\
\hline$S_{\mathrm{GBP}}$ & 0.9957 & 0.9810 & 0.4428 & 0.9992 & 0.9628 & 0.9085 \\
\hline$S_{\mathrm{GBP}}^{\prime}$ & 0.9984 & 0.9937 & 0.8856 & 0.9997 & 0.9888 & 0.9758 \\
\hline
\end{tabular}
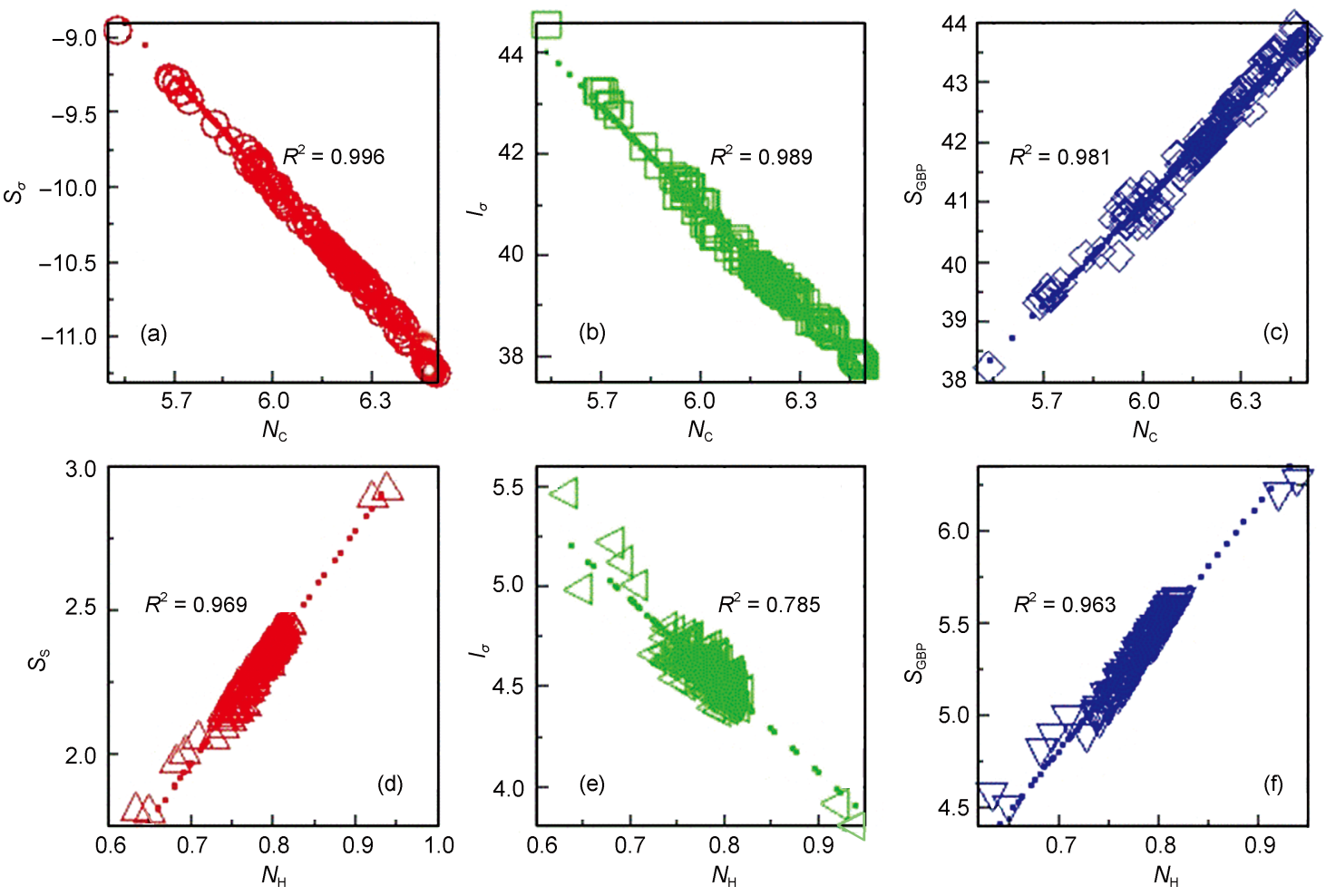

Fig.1 Scaling results for (a-c) carbon and (d-f) hydrogen atoms in molecules results with Becke's fuzzy partitioning approach for 192 carbon atoms and 252 hydrogen atoms in $\mathbf{4 2}$ molecular systems studied

Reprinted with permission from Ref.19. Copyright 2015 Royal Society of Chemistry.

opted, the steric energy can be partitioned at both the atomic and functional group levels. Its corresponding steric potential, steric charge, and steric force have been defined and evaluated for both molecules and solids ${ }^{46,55}$.

This new quantification has been applied to a number of systems and chemical phenomena ${ }^{46-58}$, such as conformational changes of small molecules, $\mathrm{S}_{\mathrm{N}} 2$ reactions, chained and branched alkanes, water clusters, anomeric effect, weak interactions, orbital interactions, and other systems. Reasonably good trends and linear relationships between theoretical result and experimental scales by Taft have recently been observed at both group and entire molecular levels.

In particular, a clear picture has emerged from these studies in conformation changes ${ }^{57}$, showing that for all systems con- sidered thus far, the electrostatic interaction plays the predominant role, whereas other effects such as steric and exchangecorrelation interactions play minor but indispensable roles. As an example, Fig. 3 shows the energetic profiles of the (a) total energy and (b) exchange-correlation energy differences with respect to the $360^{\circ}$ flexible rotation with the step size of $5^{\circ}$ for each of the six different single bonds, $\mathrm{C}-\mathrm{C}, \mathrm{C}-\mathrm{N}, \mathrm{C}-\mathrm{O}$, $\mathrm{N}-\mathrm{N}, \mathrm{N}-\mathrm{O}$, and $\mathrm{O}-\mathrm{O}$ for six simple molecules $\mathrm{CH}_{3} \mathrm{CH}_{3}$, $\mathrm{CH}_{3} \mathrm{NH}_{2}, \mathrm{CH}_{3} \mathrm{OH}, \mathrm{NH}_{2} \mathrm{NH}_{2}, \mathrm{NH}_{2} \mathrm{OH}$, and $\mathrm{H}_{2} \mathrm{O}_{2}$, respectively. As can be seen from the figure, these energy profiles are vastly different from one another in both barrier heights and curve shapes $^{57}$.

From the energy component analysis viewpoint, as shown in Fig.4, however, a much simpler picture is available. Our results 

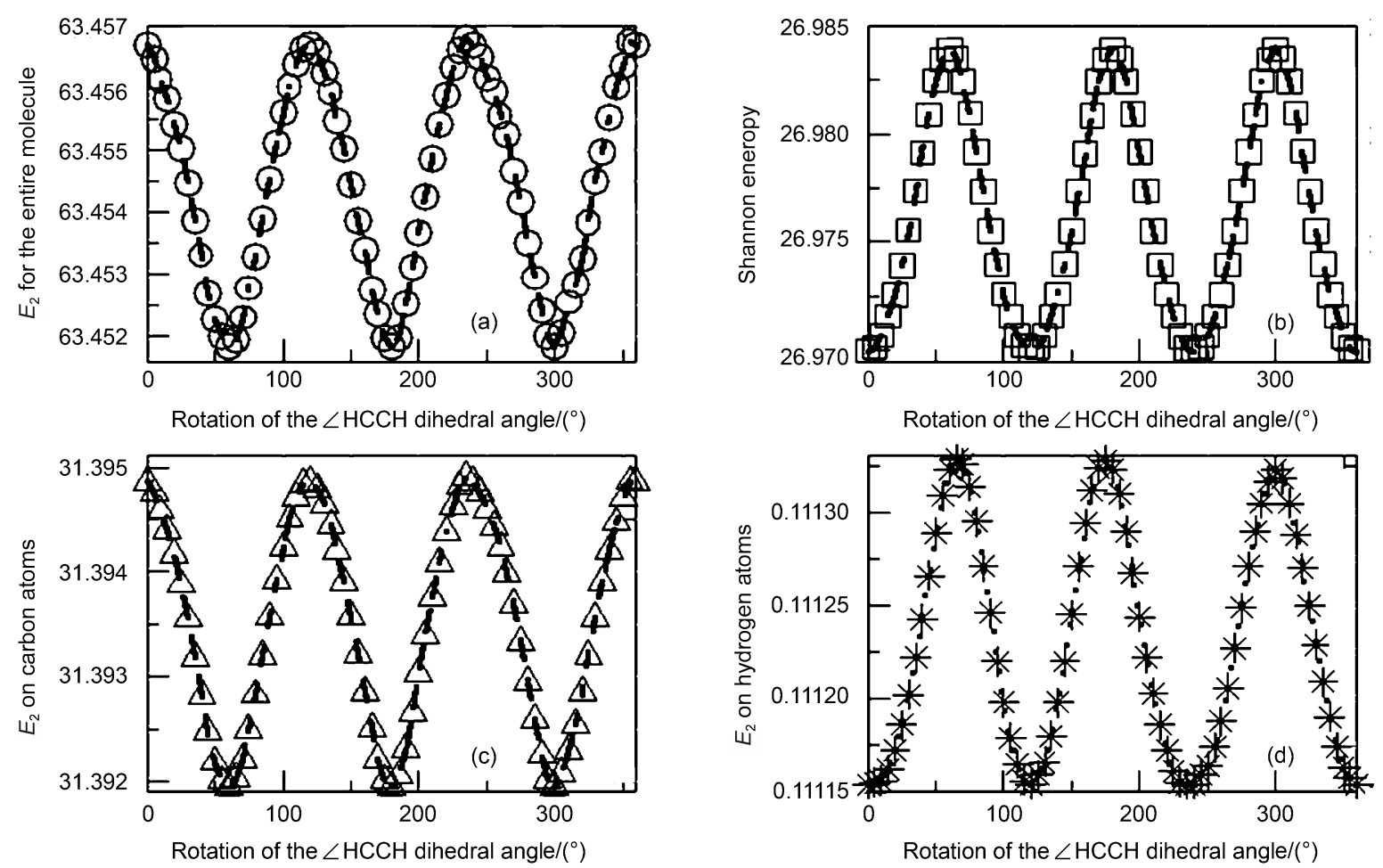

Fig.2 Profiles of information-theoretic quantities as a function of the HCCH dihedral angle for the ethane molecule Reprinted with permission from Ref.65. Copyright 2015 Chinese Chemical Society.
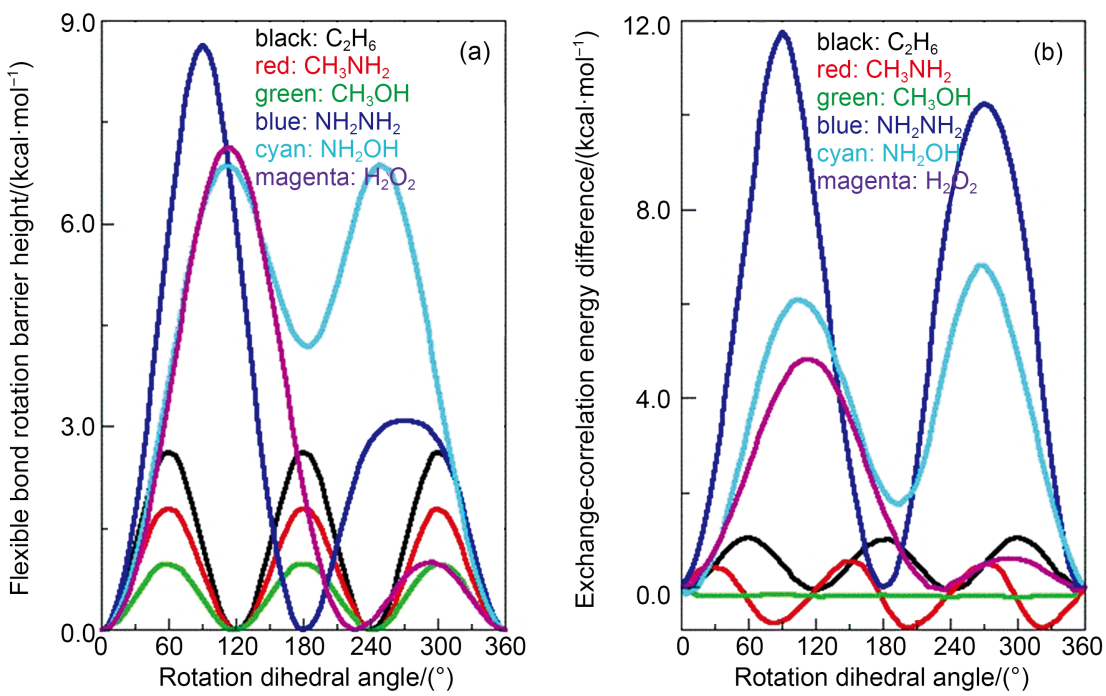

Fig.3 Profiles of (a) total energy and (b) exchange-correlation energy differences of six molecules

Reprinted with permission from Ref.57. Copyright 2013 American Chemical Society. $1 \mathrm{cal}=4.1868 \mathrm{~J}$

unambiguously show that the electrostatic interaction is the dominant contributor to the rotation barrier height. The higher the barrier, the larger the electrostatic interaction. On the other hand, our results also confirm and consolidate the earlier explanations in the literature, where both steric repulsion and hyperconjugation (quantum) effects were employed to validate the rotation barriers. As can be seen from Figs.4b and 4c, though with a smaller coefficient, both steric effect and exchange- correlation interactions positively contribute to the rotation barrier, suggesting that the higher the bond rotation barrier, the stronger the steric repulsion and the smaller the quantum effect due to exchange-correlation interactions ${ }^{57}$.

\section{Minimum information gain principle}

In 2000, Nalewajski and Parr $^{13}$ started the era of employing a simple density functional to quantify properties related to mo- 

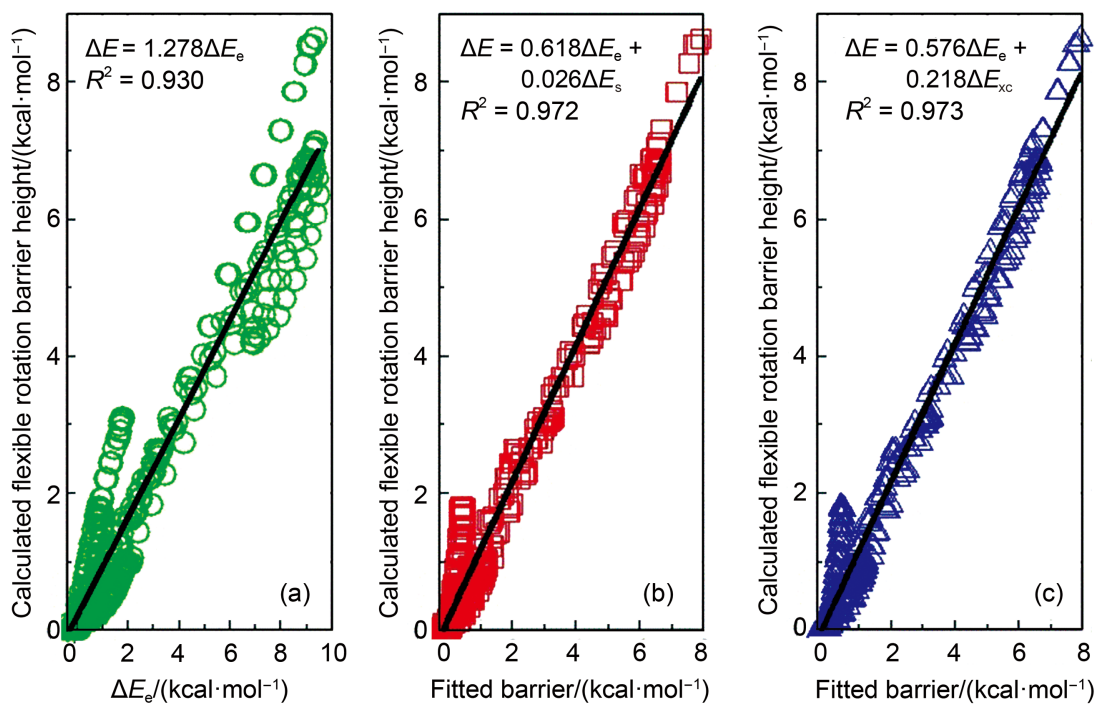

Fig.4 Least-square fittings with one and two energy components all six molecules

Reprinted with permission from Ref.57. Copyright 2013 American Chemical Society. 1 cal $=4.1868 \mathrm{~J}$

lecular structure and reactivity. In their original work, they employed the relative Shannon entropy from the information-theoretic approach, Eq.(43), which is sometimes also called the information gain, Kullback-Leibler divergence, information divergence, missing information, or entropy deficiency. From it, they derived the Hirshfeld partition of atoms in molecules, and thus the Hirshfeld charge, with the so-called minimum information gain principle.

Rewriting Eq.(43) as the information gain using atomic density

$$
I_{\mathrm{G}}=\sum_{\mathrm{A}} \int \rho_{\mathrm{A}} \ln \frac{\rho_{\mathrm{A}}}{\rho_{\mathrm{A}}^{0}} \mathrm{~d} \boldsymbol{r}
$$

where $\rho_{\mathrm{A}}$ is the electron density on atom (or group) A in a molecule, whose total electron density is $\rho$ and the total number of electrons is $N$, and $\rho_{\mathrm{A}}^{0}$ is the counterpart of atom (or group) A in the reference state, which can be neutral atom, or ion, or group, etc.

Minimizing the information gain, subject to the condition that atomic densities are always normalized to the total electron N

$$
\int \rho \mathrm{d} \boldsymbol{r}=\sum_{\mathrm{A}} \int \rho_{\mathrm{A}} \mathrm{d} \boldsymbol{r}=\sum_{\mathrm{A}} \int \rho_{\mathrm{A}}^{0} \mathrm{~d} \boldsymbol{r}=N
$$

leads to

$$
\delta\left\{I_{\mathrm{G}}-\lambda\left[\sum_{\mathrm{A}} \int_{\rho_{\mathrm{A}}}(\boldsymbol{r}) \mathrm{d} \boldsymbol{r}-N\right]\right\}=0
$$

where $\lambda$ is the Lagrange multiplier. Functional differentiation of Eq.(66) with respect to all $\rho_{\mathrm{A}}$, after some algebraic manipulations, yields that for all atoms $\mathrm{A}$, we have $\mathrm{e}^{14}$

$$
\rho_{\mathrm{A}}=\frac{\rho_{\mathrm{A}}^{0}}{\sum_{\mathrm{A}} \rho_{\mathrm{A}}^{0}} \rho
$$

This is the well-known "stockholder partition" of the electron density for atoms in molecules first proposed by Hirshfeld.

That is to say, if one employs the Hirshfeld scheme to partition atoms in a molecule, the information gain due to the formation of the molecule from the composing pieces will be minimal. In other words, atoms in molecules partitioned in this manner will preserve their identity (e.g. electrophilic and nucleophilic properties, etc.) of the reference state as much as possible. This nature of minimal information deficiency is the essence of the minimum information gain principle.

The milestone of this seminal work by Nalewajski and Parr is the fact that it serves as the first example to quantify a reactivity property of molecules with a density functional from the information-theoretic approach. Moreover, since charge is not associated with any physical observable, it has no unique definition. There are many ways to quantify charge in the literature. This work provides the first example to derive charges from a physiochemical principle.

In the next sections, besides rationalizing the validity of the Hirshfeld charge, we will demonstrate how this principle can be utilized to quantify other reactivity properties such as regioselectivity, electrophilicity, and nucleophilicity. ${ }^{16}$

\section{Information conservation principle}

Very recently, we expanded the context of the above principle, providing an in-depth understanding on the origin and nature of a few reactivity properties such as regioselectivity, electrophilicity, and nucleophilicity. ${ }^{16}$ The expansion was based on a special case of the above principle, which was called the information conservation principle.

According to the above principle, atoms in molecules tend to keep their identity of their reference state as much as possible, indicating that $\rho_{\mathrm{A}}$ and $\rho_{\mathrm{A}}^{0}$ should be similar and the difference 
between the two densities should be simulated by a perturbation expansion using the Taylor series. We can define a new variable, $x=\left(\rho_{\mathrm{A}}-\rho_{\mathrm{A}}^{0}\right) / \rho_{\mathrm{A}}$ so the information gain in Eq.(64) becomes

$$
I_{\mathrm{G}}=\sum_{\mathrm{A}} \int \rho_{\mathrm{A}} \ln \frac{1}{1-x} \mathrm{~d} \boldsymbol{r}
$$

Since $x$ is expected to be small, using $\ln \frac{1}{1-x} \approx x$ as the firstorder approximation, we have

$$
I_{\mathrm{G}} \approx \sum_{\mathrm{A}} \int\left(\rho_{\mathrm{A}}-\rho_{\mathrm{A}}^{0}\right) \mathrm{d} \boldsymbol{r}=-\sum_{\mathrm{A}} q_{\mathrm{A}}
$$

where $q_{\mathrm{A}}$ is the Hirshfeld charge on atom (or group) A. This result shows that under the first-order approximation, the information gain simply gives rise to the Hirshfeld charge distribution.

At the meanwhile, since $\rho_{\mathrm{A}}$ and $\rho_{\mathrm{A}}^{0}$ satisfy the same normalization condition, Eq.(65), the total information gain in Eq.(69) must vanish ${ }^{16}$,

$$
I_{\mathrm{G}} \approx-\sum_{\mathrm{A}} q_{\mathrm{A}} \equiv 0
$$

suggesting that under the first-order approximation, the information before and after a system is formed should be conserved. We call this result the information conservation principle. This principle, stemmed from the above first-order approximation, is a special case of the minimum information gain principle, Eq.(64), with no information gained at all. The actual value of information gain in Eq.(64) should come from the second and other higher order terms in the Taylor expansion in Eq.(68), with $\ln \frac{1}{1-x} \approx x+\frac{x^{2}}{2}+\frac{x^{3}}{3}+\cdots$.

To verify the validity of the first-order approximation introduced in Eq.(68), shown in Fig.5 is the comparison of the two

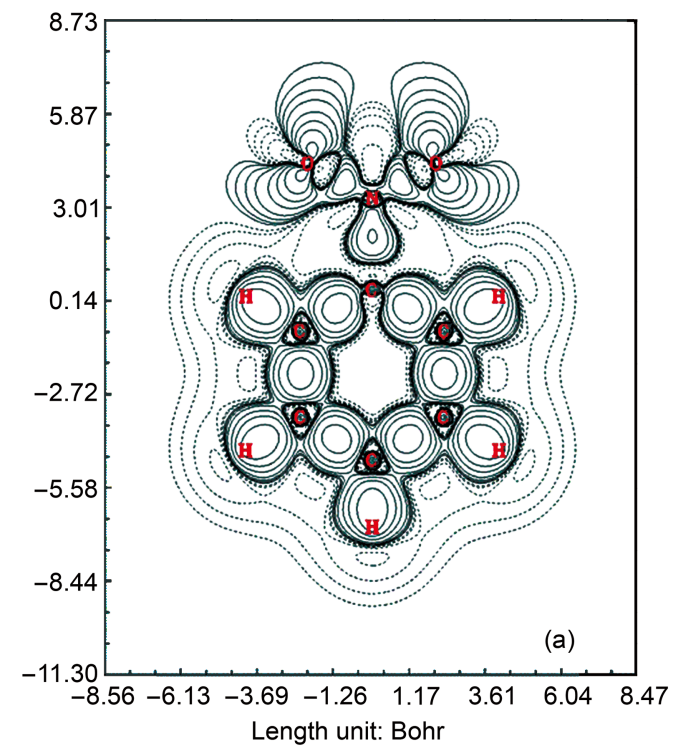

quantities without (Fig.5(a)) and with (Fig.5(b)) the approximation for the nitrobenzene molecule. As one can see from the two plots, no discernible difference can be seen from the two, and thus it confirms its validity ${ }^{16}$.

According to this principle, when a new molecular system is formed from its components, the identity of its ingredients will be preserved, at least to the first-order approximation. That is to say, if a component is electrophilic or nucleophilic in nature, it will still be so in the newly formed system as well. Moreover, to preserve the identity of the components in the new system, they will have to adjust themselves in such a manner that each of the components becomes charged according to its stockholder contribution in the electron density, as shown in Eq.(67). Put together, the new understanding from the information conservation principle proposed in this work will provide a novel approach in quantifying reactivity properties, such as electrophilicity, nucleophilicity, and regioselectivity, as will be illustrated in the following section by a number of different molecular systems ${ }^{16-22}$.

\section{Nucleophilicity and electrophilicity}

As two of the most widely used concepts in chemistry ${ }^{89-91}$, electrophilicity and nucleophilicity measure the ability of an electrophile and nucleophile to accept and donate electrons, respectively. Related to these reactivity properties is the concept of regioselectivity, on which atom or atoms these electrophilic or nucleophilic transformations are most likely to take place. A unified effort towards a general scale of nucleophilicity and electrophilicity using experimental rate constants has recently been introduced by Mayr and coworkers ${ }^{92-94}$. Theoretically, with the Fukui function from conceptual DFT one can predict the reactive site of electrophilic and nucleophilic attacks ${ }^{1,29}$. Neverthe-

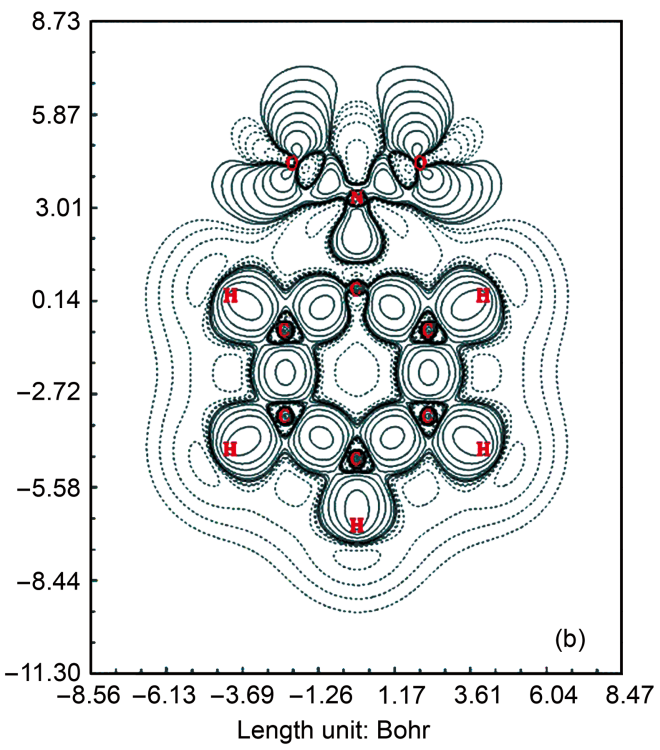

Fig.5 Validity check of the first-order approximation for information gain: (a) information gain in Eq.(64) and (b) deformation density in Eq.(69) for 
less, any effort to provide a unified theoretical framework to simultaneously quantify electrophilicity and nucleophilicity and at the same time determine their regioselectivity has been unsuccessful until recently.

In principle, the property of electrophilicity and nucleophilicity should be closely related to the lack and excess of electrons, respectively, at different atoms in molecules. Therefore, the conventional chemical wisdom that information of the atomic charge distribution in a molecule should suffice in quantifying these properties must still be valid. That is, a negatively charged atom in molecules is able to donate electrons so it should be nucleophilic in nature, whereas a positively charge atom is capable of accepting electrons so it be electrophilic. Our recent work has demonstrated that it is indeed the cas $\mathrm{e}^{16}$. Based on the principles introduced above, we employed the Hirshfeld charge for the purposes and demonstrated that regioselectivity, electrophilicity and nucleophilicity can all simultaneously be determined.

As illustrative examples, we studied 21 electrophilic systems (Scheme 1) and 22 nucleophilic molecules (Scheme 2), and compared their experimental scale of electrophilicity/nucleophilicity with both information gain and Hirshfeld charge. Figs. 6 and 7 display those comparisons, from which we find that remarkable agreement between Mayr's experimental scales and our theoretical measures can be achieved ${ }^{16}$.

In another recent work, we applied the idea to nitrogen-containing systems ${ }^{17}$. Five different categories of compounds were studied, including benzenediazonium, azodicarboxylate, diazo,

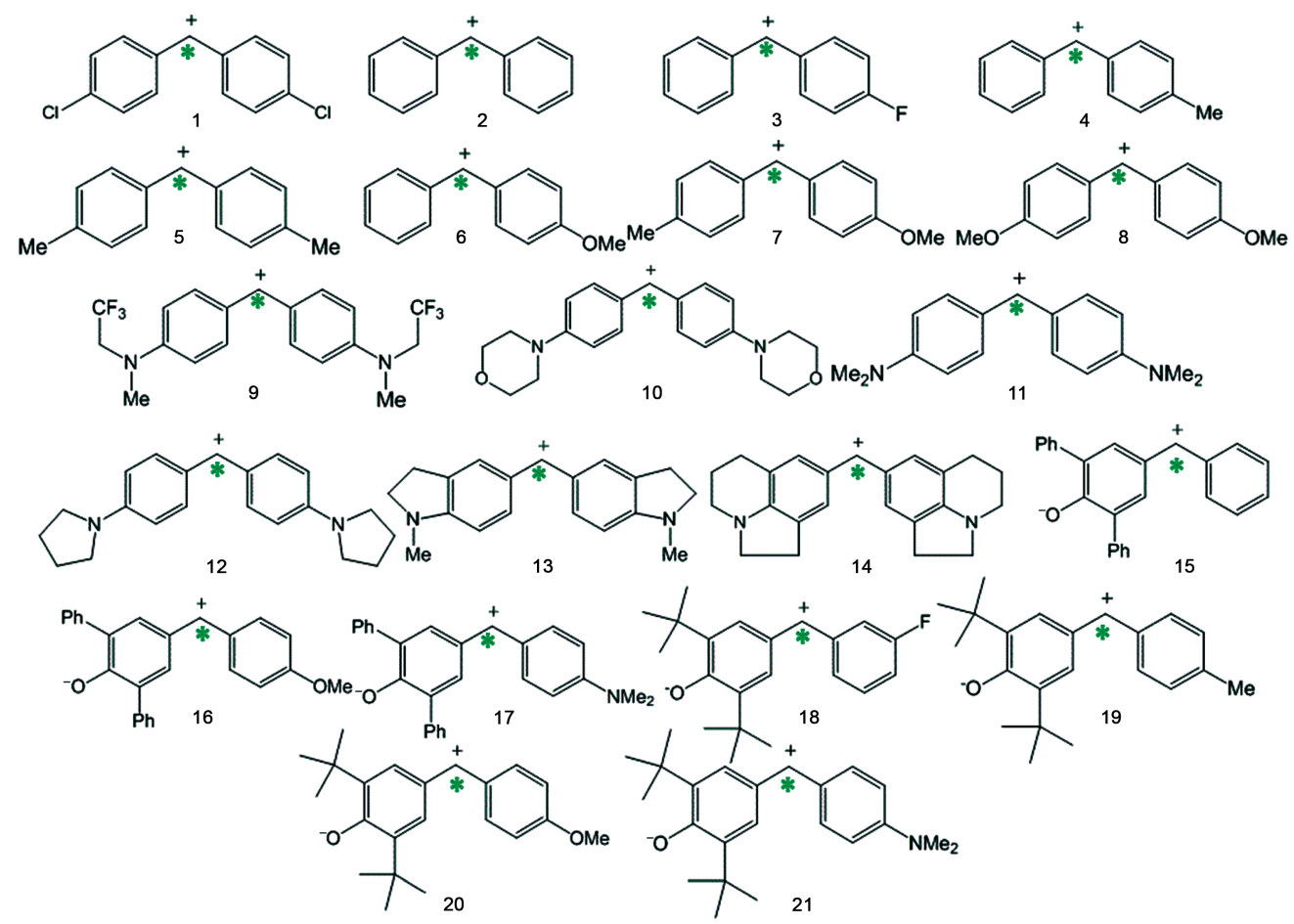

Scheme 1 Twenty-one electrophilic molecules studied

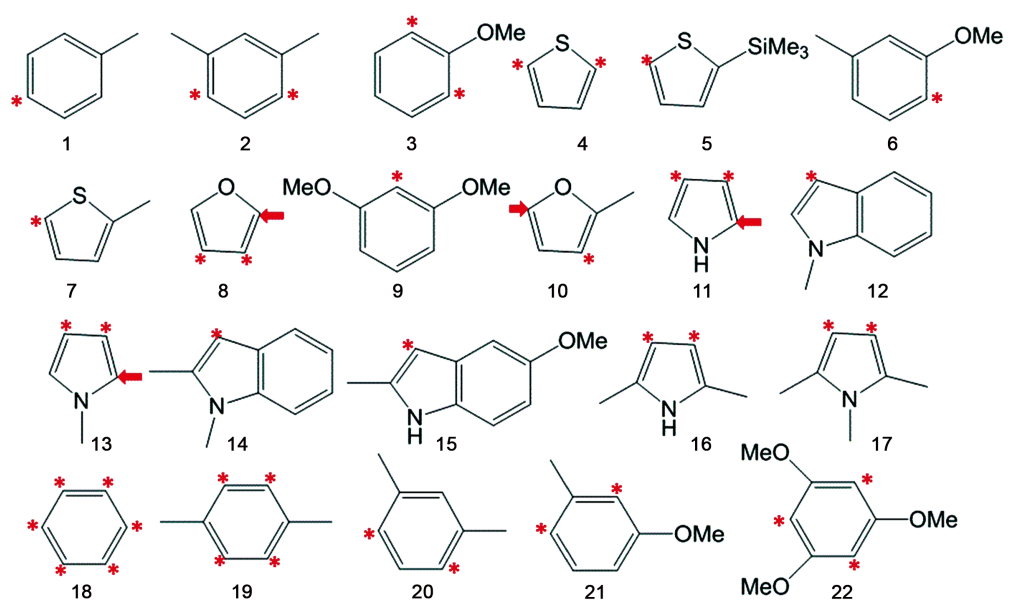

Scheme 2 Twenty-two nucleophilic molecules studied 

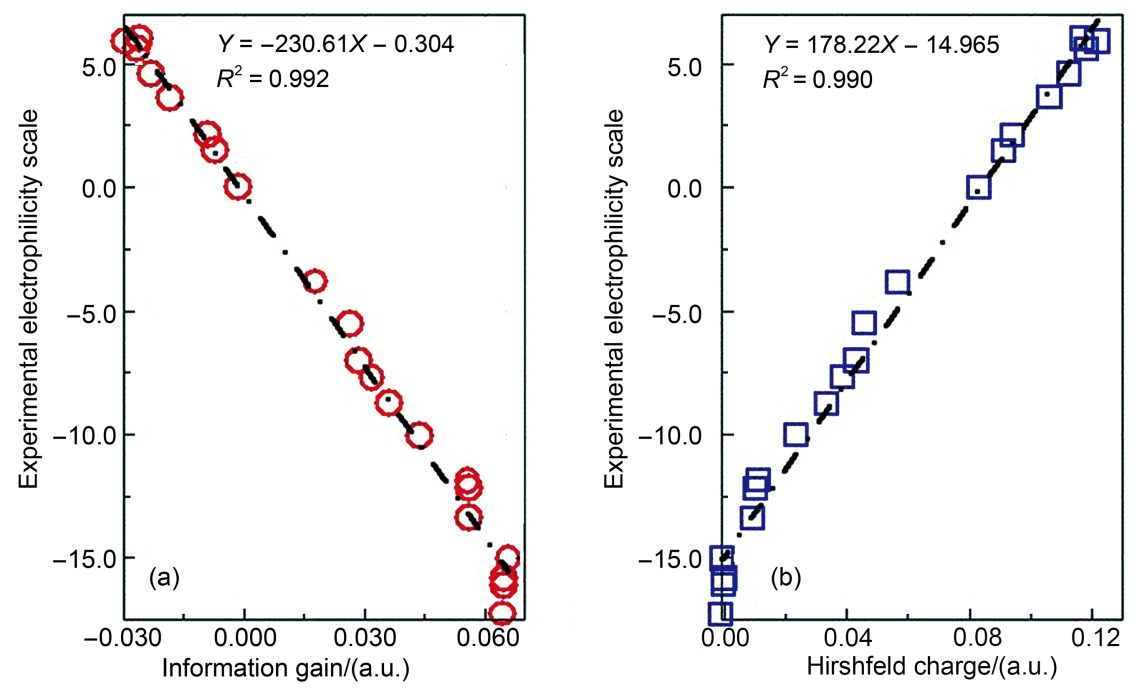

Fig.6 Comparison of the experimental electrophilicity scale of Mayr et al. with (a) information gain and (b) Hirshfeld charge for 21 systems listed in Scheme 1

Reprinted with permission from Ref.16. Copyright 2014 American Chemical Society.
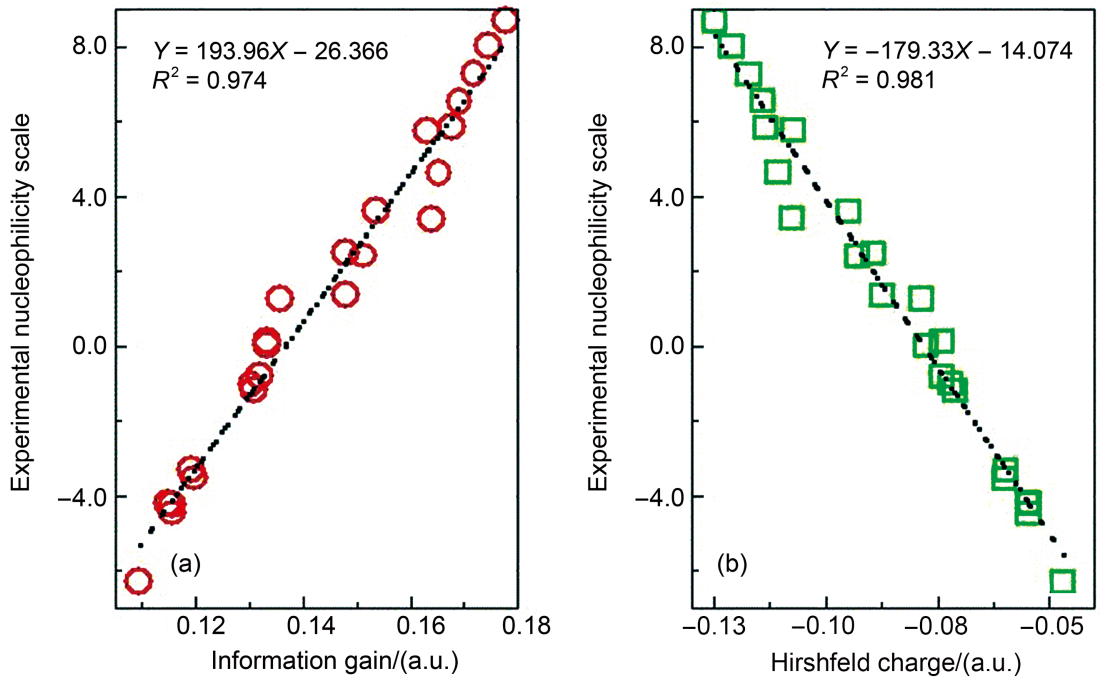

Fig.7 Comparison of the experimental nucleophilicity scale by Mayr et al. with (a) information gain and (b) Hirshfeld charge for 22 systems listed in Scheme 2

Reprinted with permission from Ref.16. Copyright 2014 American Chemical Society.

primary and secondary amines, with a total of 40 molecules. Our results show that there still exist strong linear correlations between the Hirshfeld charge and their experimental scales of electrophilicity and nucleophilicity. However, these correlations depend on the valence state and bonding environment of the nitrogen element. The linear relationship only holds true within the same category of compounds.

In addition, we separately studied electrophilic aromatic substitution $^{18,20,21}$ and bimolecular nucleophilic substitution $\left(\mathrm{S}_{\mathrm{N}} 2\right)$ reactions $^{22}$ to quantitatively examine their nucleophilicity and electrophilicity, respectively, by comparing information gain or Hirshfeld charge at the reactive center with the reaction barrier height obtained from transition states. Again, remarkably strong correlations between these quantities have been obtained. These and other results unambiguously show that information gain and Hirshfeld charge are reliable descriptors to accurately determine nucleophilicity and electrophilicity at the same time for different systems.

Closely related to nucleophilicity and electrophilicity is the chemical concept of regioselectivity, which is simply the preferred reactive center for nucleophilic or electrophilic reactions. A nucleophilic attach should take place on the atom or atoms with the most negative charge, whereas an electrophilic attach is most likely to occur at the atom or atoms with the most positive charge. The validity of our prediction with the regioselectivity has been demonstrated in the studies present above. A more interesting example on regioselectivity is the para/ortho and meta group directing effects in electrophilic aromatic substitu- 
tion reactions, as will be discussed below ${ }^{18,20}$.

\section{Regioselectivity: nature of ortholpara and meta group directing}

An electrophilic aromatic substitution is a chemical transformation in which the hydrogen atom of an aromatic ring is replaced by an electrophile as a result of an electrophilic attack on the aromatic ring. Important examples of such reactions include aromatic nitration, halogenation, sulfonation, acylation, and alkylation. Electrophilic aromatic substitution reactions as one of the most fundamental chemical processes are affected by atoms or groups already attached to the aromatic ring ${ }^{95}$. The groups that promote substitution at the ortho/para or meta positions are, respectively, called ortho/para and meta directing groups, which are often characterized by their capability to donate electrons to or withdraw electrons from the ring. Though resonance and inductive effects have been employed in textbooks and in the literature to justify this phenomenon ${ }^{96,97}$, no satisfactory quantitative interpretation is available until now.
The logic to apply the information-theoretic approach to this problem is the following. When a functional group, either ortho/ para or meta directing, is placed on the benzene ring, the nucleophilic property of the benzene ring will subsequently be altered, leading to the nonhomogeneous behavior of the six carbon atoms of the substituted benzene ring in electron donation. This change of nucleophilicity, the capability of donating electrons to the incoming electrophile, of the substituted benzene ring should be the root cause of the above ortho/para and meta directing phenomenon. ${ }^{18}$

Table 5 shows the Hirshfeld charge at ortho-, meta- and para-positions for mono-substituted benzene derivatives, Ar-R, with either ortho/para or meta directing group, R. We found that (i) for ortho/para directing groups (left 4 columns in the Table), in all the cases, the most negative charged atom is on either ortho or para position, and there is no single incidence where the meta-position possesses the most negative charge. For the meta directing groups (the right 4 columns), however, for a vast majority of the systems such as $-\mathrm{CX}_{3},-\mathrm{CHO}$,

Table 5 Hirshfeld charges at ortho-, meta-, and para-positions for mono-substituted benzene derivatives Ar-R, with both ortho/para (left four columns) and meta (right four columns) directing groups $\mathbf{R}$

\begin{tabular}{|c|c|c|c|c|c|c|c|}
\hline Ortho/Para-directing & Ortho & Meta & Para & Meta-directing & Ortho & Meta & Para \\
\hline$-\mathrm{Ar}$ & $\underline{-0.0437}$ & -0.0409 & $\underline{-0.0449}$ & $-\mathrm{CBr}_{3}$ & -0.0179 & $\underline{-0.0350}$ & -0.0293 \\
\hline$-\mathrm{Cl}$ & -0.0401 & -0.0326 & -0.0412 & $-\mathrm{CCl}_{3}$ & -0.0332 & $\underline{-0.0355}$ & -0.0291 \\
\hline$-\mathrm{CO}^{-}$ & $\underline{-0.1509}$ & -0.0757 & -0.2252 & $-\mathrm{CF}_{3}$ & -0.0296 & -0.0354 & -0.0272 \\
\hline$-\mathrm{Et}$ & -0.0492 & -0.0418 & -0.0505 & $-\mathrm{CHO}$ & -0.0275 & -0.0424 & -0.0223 \\
\hline$-\mathrm{F}$ & -0.0542 & -0.0298 & -0.0522 & $-\mathrm{CN}$ & -0.0179 & -0.0334 & -0.0209 \\
\hline$-\mathrm{H}$ & -0.0426 & -0.0426 & -0.0426 & $-\mathrm{COBr}$ & -0.0099 & -0.0386 & -0.0139 \\
\hline$-i \operatorname{Pr}$ & -0.0499 & -0.0423 & $\underline{-0.0505}$ & $-\mathrm{COCl}$ & -0.0196 & -0.0394 & -0.0148 \\
\hline$-\mathrm{Me}$ & $\underline{-0.0516}$ & -0.0415 & $\underline{-0.0510}$ & $-\mathrm{COF}$ & -0.0194 & $\underline{-0.0395}$ & -0.0157 \\
\hline - NArCOH & -0.0467 & -0.0358 & $\underline{-0.0422}$ & $-\mathrm{COMe}$ & -0.0322 & $\underline{-0.0449}$ & -0.0262 \\
\hline$-\mathrm{N}(\mathrm{COCl})_{2}$ & -0.0320 & -0.0308 & -0.0255 & $-\mathrm{CONH}_{2}$ & -0.0367 & -0.0431 & -0.0285 \\
\hline$-\mathrm{N}(\mathrm{COF})_{2}$ & $\underline{-0.0328}$ & $\underline{-0.0292}$ & -0.0266 & $-\mathrm{COOAr}$ & -0.0249 & $\underline{-0.0434}$ & -0.0233 \\
\hline$-\mathrm{NCOH}_{2}$ & $\underline{-0.0369}$ & $\underline{-0.0318}$ & -0.0297 & $-\mathrm{COOH}$ & -0.0233 & $\underline{-0.0430}$ & -0.0220 \\
\hline$-\mathrm{NH}_{2}$ & $-\underline{-0.0713}$ & -0.0342 & $\underline{-0.0705}$ & $-\mathrm{COO}^{-}$ & -0.0451 & $\underline{-0.0707}$ & -0.0728 \\
\hline$-\mathrm{NHAr}$ & $\underline{-0.0598}$ & -0.0374 & $\underline{-0.0564}$ & $-\mathrm{COOMe}$ & -0.0259 & $\underline{-0.0443}$ & -0.0249 \\
\hline$-\mathrm{NHCOH}$ & $\underline{-0.0545}$ & -0.0314 & -0.0497 & $-\mathrm{COO} t \mathrm{Bu}$ & -0.0277 & $\underline{-0.0455}$ & -0.0269 \\
\hline - NHCOMe & $\underline{-0.0395}$ & -0.0371 & -0.0373 & $-\mathrm{CO} t \mathrm{Bu}$ & -0.0320 & $\underline{-0.0440}$ & -0.0307 \\
\hline$-\mathrm{NHMe}$ & $\underline{-0.0742}$ & -0.0352 & -0.0723 & $-\mathrm{H}$ & -0.0426 & -0.0426 & -0.0426 \\
\hline$-\mathrm{NH} t \mathrm{Bu}$ & -0.0490 & -0.0399 & -0.0563 & $-\mathrm{NAr}_{3}^{+}$ & -0.0321 & -0.0195 & -0.0107 \\
\hline$-\mathrm{NMe}_{2}$ & -0.0722 & -0.0369 & -0.0717 & $-\mathrm{NH}_{2} \mathrm{Ar}^{+}$ & -0.0329 & -0.0088 & -0.0031 \\
\hline$-\mathrm{N} t \mathrm{Bu}_{2}$ & -0.0375 & -0.0429 & $\underline{-0.0490}$ & $-\mathrm{NH}_{2} \mathrm{Me}^{+}$ & -0.0318 & -0.0044 & -0.0005 \\
\hline$-\mathrm{OAr}$ & $\underline{-0.0546}$ & -0.0344 & $\underline{-0.0554}$ & $-\mathrm{NH}_{2} t \mathrm{Bu}^{+}$ & $\underline{-0.0304}$ & $\underline{-0.0091}$ & -0.0042 \\
\hline$-\mathrm{OCHO}$ & -0.0465 & -0.0301 & -0.0414 & $-\mathrm{NH}_{3}^{+}$ & $\underline{-0.0288}$ & -0.0024 & 0.0033 \\
\hline - OCOMe & -0.0448 & -0.0329 & -0.0453 & $-\mathrm{NHAr}_{2}^{+}$ & $\underline{-0.0347}$ & $\underline{-0.0116}$ & -0.0082 \\
\hline$-\mathrm{OCONH}_{2}$ & $\underline{-0.0456}$ & -0.0341 & $\underline{-0.0450}$ & $-\mathrm{NHMe}_{2}^{+}$ & $\underline{-0.0328}$ & $\underline{-0.0060}$ & -0.0038 \\
\hline$-\mathrm{OCO} t \mathrm{Bu}$ & -0.0452 & -0.0333 & -0.0462 & $-\mathrm{NH} t \mathrm{Bu}_{2}^{+}$ & -0.0282 & -0.0138 & -0.0114 \\
\hline$-\mathrm{OEt}$ & $\underline{-0.0617}$ & -0.0337 & -0.0656 & $-\mathrm{NMe}_{3}^{+}$ & -0.0333 & -0.0111 & -0.0053 \\
\hline$-\mathrm{OH}$ & $\underline{-0.0747}$ & -0.0327 & -0.0647 & $-\mathrm{NO}_{2}$ & -0.0166 & $\underline{-0.0344}$ & -0.0154 \\
\hline$-\mathrm{O} i \mathrm{Pr}$ & $\underline{-0.0626}$ & -0.0339 & $\underline{-0.0667}$ & $-\mathrm{NO}$ & -0.0201 & $\underline{-0.0400}$ & -0.0200 \\
\hline$-\mathrm{O}^{-}$ & $\underline{-0.1285}$ & -0.0749 & $\underline{-0.1567}$ & $-\mathrm{SO}_{3} \mathrm{H}$ & -0.0183 & $\underline{-0.0335}$ & -0.0157 \\
\hline$-\mathrm{OMe}$ & -0.0611 & -0.0331 & $\underline{-0.0649}$ & $-\mathrm{SO}_{3}^{-}$ & -0.0331 & -0.0585 & -0.0624 \\
\hline
\end{tabular}


Table 6 Prediction of ortho/para and meta group directing for 23 mono-substituted benzene derivatives, Ar-R, in electrophilic aromatic substitution

\begin{tabular}{|c|c|c|c|c|c|c|c|}
\hline $\mathrm{R}$ & Ortho & Meta & Para & HOMO & $\Delta Q_{\mathrm{C}}$ & $\Delta Q_{\mathrm{C}_{6} \mathrm{H}_{5}}$ & Prediction \\
\hline$-\mathrm{CH}_{2}^{-}$ & -0.1260 & -0.0768 & $\underline{-0.1773}$ & -0.0295 & -0.2143 & -0.5608 & ortho/para \\
\hline$-\mathrm{GeH}_{3}$ & -0.0267 & -0.0409 & -0.0368 & -0.3387 & 0.0148 & -0.0467 & meta \\
\hline$-\mathrm{NCl}_{2}$ & $\underline{-0.0522}$ & -0.0322 & -0.0462 & -0.3304 & 0.0114 & 0.1062 & ortho/para \\
\hline$-\mathrm{NCS}$ & -0.0333 & -0.0304 & $=0.0347$ & -0.3241 & 0.0501 & 0.2230 & ortho/para \\
\hline$-\mathrm{NF}_{2}$ & -0.0404 & -0.0316 & -0.0374 & -0.3516 & 0.0377 & 0.1505 & ortho/para \\
\hline$-\mathrm{NH}^{-}$ & -0.1363 & -0.0751 & -0.1683 & -0.0607 & -0.2081 & -0.4874 & ortho/para \\
\hline$-\mathrm{NHSiH}_{3}$ & -0.0691 & -0.0326 & $\underline{-0.0706}$ & -0.2938 & -0.0123 & 0.0417 & ortho/para \\
\hline$-\mathrm{OCl}$ & -0.0653 & -0.0299 & -0.0553 & -0.3300 & 0.0192 & 0.1347 & ortho/para \\
\hline$-\mathrm{OF}$ & $\underline{-0.0590}$ & -0.0273 & $\underline{-0.0535}$ & -0.3374 & 0.0329 & 0.1565 & ortho/para \\
\hline$-\mathrm{PH}_{2}$ & -0.0287 & -0.0404 & -0.0347 & -0.3421 & 0.0168 & 0.0110 & meta \\
\hline$-\mathrm{PO}_{2}$ & 0.0082 & $\underline{-0.0347}$ & -0.0018 & -0.3743 & 0.0864 & 0.1937 & meta \\
\hline$-\mathrm{PO}$ & -0.0139 & -0.0422 & -0.0118 & -0.3587 & 0.0490 & 0.0785 & meta \\
\hline$-\mathrm{SCl}$ & -0.0496 & -0.0310 & -0.0439 & -0.3183 & 0.0193 & 0.0659 & ortho/para \\
\hline$-\mathrm{SCN}$ & -0.0281 & -0.0328 & -0.0223 & -0.3648 & 0.0475 & 0.1445 & meta \\
\hline$-\mathrm{SeH}$ & $\underline{-0.0371}$ & -0.0341 & -0.0464 & -0.3051 & 0.0188 & 0.0712 & ortho/para \\
\hline$-\mathrm{SeO}_{3} \mathrm{H}$ & -0.0012 & $\underline{-0.0310}$ & -0.0109 & -0.3749 & 0.0847 & 0.2389 & meta \\
\hline$-\mathrm{SF}$ & $\underline{-0.0517}$ & -0.0307 & -0.0453 & -0.3185 & 0.0254 & 0.0671 & ortho/para \\
\hline$-\mathrm{SH}$ & $\underline{-0.0505}$ & -0.0328 & -0.0488 & -0.3104 & 0.0121 & 0.0459 & ortho/para \\
\hline$-\mathrm{SiF}_{3}$ & -0.0166 & -0.0374 & -0.0190 & -0.3600 & 0.0430 & 0.0459 & meta \\
\hline$-\mathrm{SiH}_{3}$ & -0.0342 & $\underline{-0.0431}$ & -0.0352 & -0.3398 & 0.0032 & -0.0447 & meta \\
\hline$-\mathrm{SiHO}$ & -0.0190 & -0.0420 & -0.0135 & -0.3617 & 0.0464 & 0.0580 & meta \\
\hline$-\mathrm{SiOOH}$ & -0.0130 & $\underline{-0.0417}$ & -0.0142 & -0.3585 & 0.0500 & 0.0709 & meta \\
\hline$-\mathrm{SiOO}^{-}$ & -0.0308 & $\underline{-0.0675}$ & -0.0611 & -0.2142 & -0.0533 & -0.1604 & meta \\
\hline
\end{tabular}

$-\mathrm{CN},-\mathrm{COR},-\mathrm{NO}_{2},-\mathrm{NO}$, and $-\mathrm{SO}_{3} \mathrm{H}$, the meta position possesses the largest Hirshfeld charge, consistent with experimental evidence. The outliers are $-\mathrm{NH}_{3}^{+}$and $-\mathrm{SO}_{3}^{-}$systems, where the meta position has the second largest negative charge $^{18}$.

Using the Hirshfeld charge as an indication of the regioselectivity, we can predict the reaction centers for unknown systems. Some results are shown in Table $6^{18}$.

To provide quantitative understanding about the role of the Hirshfeld charge on the impact of ortho/para and meta group directing effect ${ }^{20,21}$, we continued the study by examining the transition state structure and activation energy of an identity reaction for a series of mono-substituted-benzene molecules reacting with hydrogen fluoride using $\mathrm{BF}_{3}$ as the catalyst in the gas phase (Scheme 3). A total of 18 substitution groups were considered, nine of which were ortho/para directing and the other nine groups meta-directing. From this study ${ }^{20,21}$, we found that the barrier height of these reactions strongly correlates with the Hirshfeld charge on the regioselective site for both ortho/para and meta-directing groups, with the correlation coefficient $R^{2}$ both better than 0.96 . We also discovered a less accurate correlation between the barrier height and HOMO energy. These results reconfirm the validity and effectiveness of employing the Hirshfeld charge as a reliable descriptor of both reactivity and regioselectivity for this vastly important category of chemical transformations. Fig. 8 exhibits some of the results from the study, confirming the close relationship between Hirshfeld charge and regioselectivity. This relationship has also recently been confirmed by $\mathrm{S}_{\mathrm{N}} 2$ reaction systems $^{22}$.

\section{Outlooks: reactivity as density functionals}

What is reactivity? Reactivity of a molecular system is the tendency of the system to undergo a chemical transformation, which should be an intrinsic property of the system determined a priori by its composition and structure. This tendency could include changing from one conformation of the molecule to an-

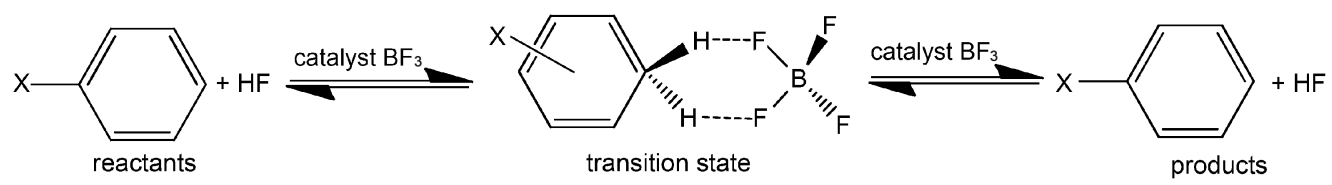

Scheme 3 Electrophilic aromatic substitution reaction studied using the identity reaction of mono-substituted-benzene molecules reacting with 

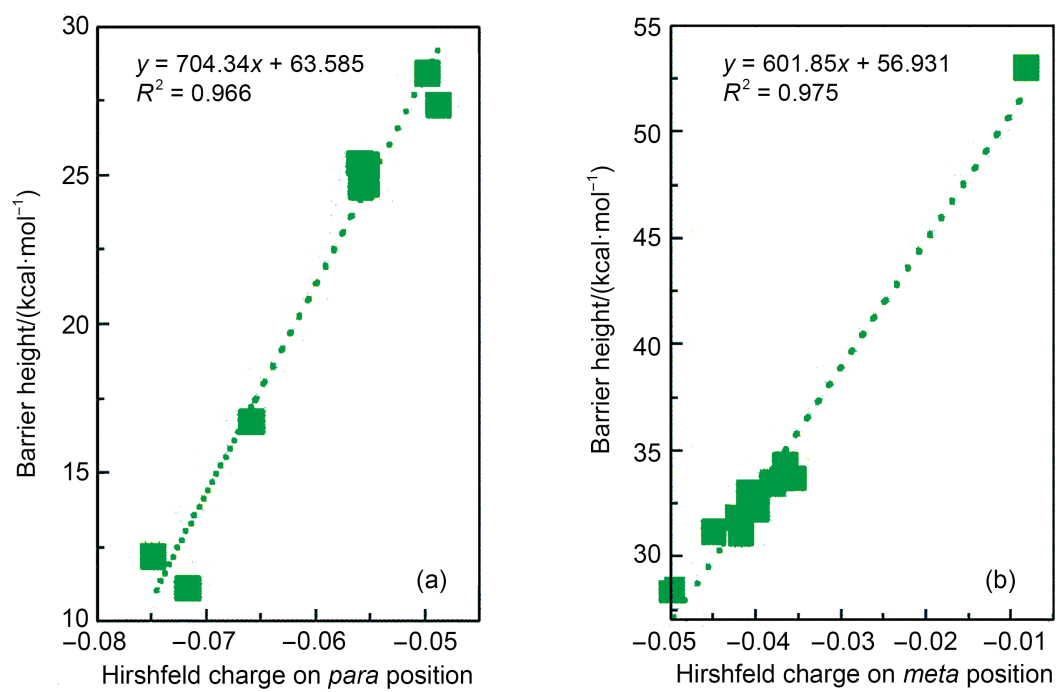

Fig.8 Strong linear correlations between Hirshfeld charge and reaction barrier height for electrophilic aromatic substation reactions with both ortho/para and meta directing groups

Reprinted with permission from Ref.20. Copyright 2015 American Chemical Society. $1 \mathrm{cal}=4.1868 \mathrm{~J}$

other one, losing or accepting one or more electrons or protons, responding to attacks by external reagents such as catalysts or external fields such as photoexcitation, among other possible changes or perturbations. Molecular orbital theory does provide us with insights using frontier orbitals. However, its main obstacle is the fact that the more accurate the computational results are, the more ambiguous their physical meanings are. This is because orbitals are human invention and thus their applicability is limited. On the other hand, one does not have to use orbitals to understand and predict molecular reactivity. DFT provides with us such a rigorous and robust alternative, where one employs electron density and its associated quantities instead of orbitals for the purpose. Earlier success in conceptual DFT with the introduction of concepts such as electronegativity, hardness, Fukui function, and dual descriptors has evidently witnessed the validity and effectiveness of such alternative tools. There exist, however, many challenges. We recently adventured a new pathway by appreciating and quantifying reactivity properties with density functionals. Key questions remaining are now the following: How should this tendency of molecular changes be described? Can it be represented by one or more density functionals? Or is it possible that each tendency of the above changes be appropriately attributed by a density functional? Keeping in mind that according to DFT, the electron density alone is adequate to determine all these properties in the ground state, we are confident that such a proposition is not completely unjustified.

Indeed, above results from the recent literature demonstrated the validity of such an effort to describe structure and reactivity properties using density functionals. Steric effect quantified using the Weizsäcker kinetic energy density functional is one example. Electrophilicity, nucleophilicity, and regioselectivity in terms of information gain or Kullback-Leibler divergence is an- other one, which enables us to provide a completely new understanding about the ortho/para/meta group directing phenomena for electrophilic aromatic substitution reactions. The density functionals we discussed in this review not only are simple in their forms, but also originate from the information theory. They possess amiable scaling properties, and because of the nature of the electron density, many of these quantities are inter-correlated. In addition, numerous principles have been established in the literature, such as the principle of extreme physical information, minimum information gain principle, and information conservation principle. These works should serve as the foundation of the information-theoretic approach in density functional reactivity theory.

So, what is next? We can answer this question by proceeding in two pathways. From the theoretical development point of view, there are a number of topics that we can quickly pick up and pursue in the near future. At first, the Kullback-Leibler divergence is the relative Shannon entropy. How does it differ from the relative Rényi entropy? Are they somehow related? Secondly, what are the local behaviors of steric/quantum potential, steric/quantum charge, and other local functions and quantities stemmed from the information-theoretic approach? What are their roles in determining reactivity and selectivity for chemical reactions? More importantly, how can we go beyond the information-theoretic approach and systematically develop different density functionals to address different needs in predicting and understanding different molecular structure and reactivity properties?

From the viewpoint of structure and reactivity properties, acidity/basicity and redox potentials are the two extremely important intrinsic physiochemical properties of molecules that require a lot of our attentions. Earlier, we proposed to employ the molecular electrostatic potential on the nucleus of the acidic or 
basic atom or its valence natural atomic orbital energy as an accurate descriptor of the acidity or basicity ${ }^{31-35}$. This work however has its limitations. That is, different systems tend to have different correlations so any effort to put different categories of molecules into one scaling system has failed miserably. We observed the same issue with electrophilicity and nucleophilicity scales. Are these phenomena related? Is it ever possible to come up with a universal scale for acidity/basicity and electrophilicity/nucleophilicity? Finally, with reliable tools dealing with regioselectivity and electrophilicity/nucleophilicity available in the literature, we are in a better position than ever to tackle the problems related to stereoselectivity, enantioselectivity, diasteroselectivity, and other chiral or asymmetric chemistry related topics.

To wrap up, it is our humble but cautiously optimistic belief that a brand new door to appreciate and entertain molecular reactivity from the perspective of density functional reactivity theory has just opened. A bright future lies ahead. Opportunities are abundant.

Acknowledgment: The author is indebted immensely to many of his former and present students and worldwide collaborators in the last few years. In particular, he is grateful to Dr. LU Tian of Beijing Kein Research Center for Natural Sciences for coding all the formulas in the text in the MultiWFN package, and to Professor PARR Robert G of University of North Carolina at Chapel Hill for constant attention, discussion, and encouragement. The author also wishes to express his sincere gratitude to Professor Emeritus LI Run-E of Hunan Normal University, Changsha, for her stimulation of the author's interest in molecular reactivity when he took the introductory organic chemistry 32 years ago taught by Professor LI. The author acknowledges support from the Research Computing Center, University of North Carolina at Chapel Hill for accessing computing facilities.

\section{References}

(1) Parr, R. G.; Yang, W. Density-Functional Theory of Atoms and Molecules. In International Series of Monographs on Chemistry; Clarendon Press: Oxford, England, 1989.

(2) Levy, M.; Ouyang, H. Phys. Rev. A 1988, 38, 625. doi: 10.1103/PhysRevA.38.625

(3) Herring, C.; Chopra, M. Phys. Rev. A 1988, 37, 311.

(4) Liu, S. B.; Ayers, P. W. Phys. Rev. A 2004, 70, 022501. doi: 10.1103/PhysRevA.70.022501

(5) Geerlings, P.; DeProft, F.; Langenaeker, W. Chem. Rev. 2003, 103, 1793. doi: 10.1021/cr990029p

(6) Chattaraj, P. K.; Sarkar, U.; Roy, D. R. Chem. Rev. 2006, 106, 2065. doi: 10.1021/cr040109f

(7) Liu, S. B. Acta Phys. -Chim. Sin. 2009, 25, 590. [刘述斌. 物理化 学学报, 2009, 25, 590.] doi: 10.3866/PKU.WHXB20090332

(8) Fukui, K. Accounts Chem. Res. 1971, 4, 57. doi: 10.1021/ar50038a003

(9) Hoffmann, R.; Woodward, R. B. J. Am. Chem. Soc. 1965, 87, 2046. doi: 10.1021/ja01087a034

(10) Geerlings, P.; Ayers, P. W.; Toro-Labbé, A.; Chattaraj, P. K.; De Proft, F. Accounts Chem. Res. 2012, 45, 683. doi: 10.1021/ar200192t

(11) Bader, R. F. W. Atoms in Molecules: A Quantum Theory; Oxford University Press: Oxford, England, 1990.

(12) Johnson, E. R.; Keinan, S.; Mori-SÁnchez, P.; Contreras-García, J.; Cohen, A. J.; Yang, W. J. Am. Chem. Soc 2010, 132, 6498.

(13) Nalewajski, R. F.; Parr, R. G. Proc. Natl. Acad. Sci. U. S. A. 2000, 97, 8879. doi: 10.1073/pnas.97.16.8879

(14) Hirshfeld, F. Theor. Chim. Acc. 1977, 44, 129. doi: 10.1007/BF00549096

(15) Liu, S. B. J. Chem. Phys. 2007, 126, 244103. doi: 10.1063/1.2747247

(16) Liu, S. B.; Rong, C. Y.; Lu, T. J. Phys. Chem. A 2014, 118, 3698. doi: 10.1021/jp5032702

(17) Zhou, X. Y.; Rong, C. Y.; Lu, T.; Liu, S. B. Acta Phys. -Chim. Sin. 2014, 30, 2055. [周夏禹, 荣春英, 卢 天, 刘述斌. 物理化 学学报, 2014, 30, 2055.] doi: 10.3866/PKU.WHXB201409193

(18) Liu, S. B. J. Chem. Phys. 2014, 141, 194109. doi: 10.1063/1.4901898

(19) Rong, C. Y.; Lu, T.; Ayers, P. W.; Chattaraj, P. K.; Liu, S. B. Phys. Chem. Chem. Phys. 2015, 17, 4977; Phys. Chem. Chem. Phys. 2015, 17, 11110.

(20) Liu, S. B. J. Phys. Chem. A 2015, 119, 3107. doi: 10.1021/acs.jpca.5b00443

(21) Wu, W. J.; Wu, Z. M., Rong, C. Y.; Lu, T.; Huang, Y.; Liu, S. B. J. Phys. Chem. A 2015, 119, 8216. doi: 10.1021/acs.jpca.5b04309

(22) Wu, W. Z.; Rong, C. Y.; Lu, T.; Ayers, P. W.; Liu, S. B. Phys. Chem. Chem. Phys. 2015,17, 27052. doi: 10.1039/C5CP04442A

(23) Nagy, Á. Int. J. Quantum Chem. 2015, 115, 1392. doi: 10.1002/qua.v115.19

(24) Nagy, Á. Europhys. Lett. 2015, 109, 60002. doi: 10.1209/02955075/109/60002

(25) Nagy, Á.; Romera, E. Chem. Phys. Lett. 2014, 597, 139. doi: 10.1016/j.cplett.2014.02.032

(26) Nagy, Á. Int. J. Quantum Chem. 2014, 114, 24812.

(27) Nagy, Á.; Romera, E. Chem. Phys. Lett. 2010, 490, 242. doi: 10.1016/j.cplett.2010.03.057

(28) Nagy, Á.; Romera, E. Int. J. Quantum Chem. 2009, 109, 2490. doi: 10.1002/qua.v109:11

(29) Parr, R. G.; Yang, W. J. Am. Chem. Soc. 1984, 106, 4049. doi: 10.1021/ja00326a036

(30) Morell, C.; Grand, A.; Toro-Labbé, A. J. Phys. Chem. A 2005, 109, 205. doi: 10.1021/jp046577a

(31) Liu, S. B.; Pedersen, L. G. J. Phys. Chem. A 2009, 113, 3648. doi: $10.1021 / j p 811250 \mathrm{r}$

(32) Liu, S. B.; Schauer, C. K.; Pedersen, L. G. J. Chem. Phys. 2009, 131, 164107. doi: 10.1063/1.3251124

(33) Burger, S. K.; Liu, S. B.; Ayers, P. W. J. Phys. Chem. A 2011, 
115, 1293. doi: 10.1021/jp111148q

(34) Huang, Y.; Liu, L.; Liu, W.; Liu, S. G.; Liu, S. B. J. Phys. Chem. A 2011, 115, 14697. doi: 10.1021/jp209540p

(35) Huang, Y.; Liu, L.; Liu, S. B. Chem. Phys. Lett. 2012, 527, 73. doi: 10.1016/j.cplett.2012.01.014

(36) Liu, S. B.; Ess, D. H.; Schauer, C. K. J. Phys. Chem. A 2011, 115, 4738. doi: 10.1021/jp112319d

(37) Kumar, N.; Liu, S. B.; Kozlowski, P. M. J. Phys. Chem. Lett. 2012, 3, 1035.

(38) Pan, S.; Sola, M.; Chattaraj, P. K. J. Phys. Chem. A 2013, 117, 1843. doi: 10.1021/jp312750n

(39) Chattaraj, P. K.; Giri, S.; Duley, S. J. Phys. Chem. A 2012, 116, 790. doi: 10.1021/jp208541x

(40) von Szentpaly, L. J. Phys. Chem. A 2011, 115, 8528.

(41) von Szentpaly, L. J. Phys. Chem. A 2013, 117, 200. doi: 10.1021/jp3103386

(42) von Szentpaly, L. J. Phys. Chem. A 2015, 119, 1715. doi: 10.1021/jp5084345

(43) Nalewajski, R. F.; Parr, R. G. J. Phys. Chem. A 2001, 105, 7391. doi: 10.1021/jp004414q

(44) Parr, R. G.; Ayers, P. W.; Nalewajski, R. F. J. Phys. Chem. A 2005, 109, 3957. doi: 10.1021/jp0404596

(45) Ayers, P. W. Theor. Chem. Acc. 2006, 115, 370. doi: 10.1007/s00214-006-0121-5

(46) Tsirelson, V. G.; Stash, A. I.; Liu, S. B. J. Chem. Phys. 2010, 133, 114110. doi: 10.1063/1.3492377

(47) Liu, S. B. J. Chem. Phys. 2007, 126, 191107. doi: $10.1063 / 1.2741244$

(48) Esquivel, R. O.; Liu, S. B.; Angulo, J. C.; Dehesa, J. S.; Antolín, J.; Molina-Espíritu, M. J. Phys. Chem. A 2011, 115, 4406. doi: 10.1021/jp1095272

(49) Liu, S. B.; Govind, N. J. Phys. Chem. A 2008, 112, 6690. doi: 10.1021/jp800376a

(50) Liu, S. B.; Govind, N.; Pedersen, L. G. J. Chem. Phys. 2008, 129, 094104. doi: $10.1063 / 1.2976767$

(51) Liu, S. B.; Hu, H.; Pedersen, L. G. J. Phys. Chem. A 2010, 114, 5913. doi: 10.1021/jp101329f

(52) Ess, D. H.; Liu, S. B.; De Proft, F. J. Phys. Chem. A 2010, 114, 12952. doi: 10.1021/jp108577g

(53) Huang, Y.; Zhong, A. G.; Yang, Q.; Liu, S. B. J. Chem. Phys. 2011, 134, 084103. doi: 10.1063/1.3555760

(54) Zhao, D. B.; Rong, C. Y.; Jenkins, S.; Kirk, S. R.; Yin, D. L.; Liu, S. B. Acta Phys. -Chim. Sin. 2013, 29, 43. [赵东波, 荣春英, 苏 曼, 苏 文, 尹笃林, 刘述斌. 物理化学学报, 2013, 29, 43.] doi: 10.3866/PKU.WHXB201211121

(55) Tsirelson, V. G.; Stash, A. I.; Karasiev, V. V.; Liu, S. B. Comp. Theor. Chem. 2013, 1006, 92. doi: 10.1016/j.comptc.2012.11.015

(56) Torrent-Sucarrat, M.; Liu, S. B.; De Proft, F. J. Phys. Chem. A 2009, 113, 3698. doi: 10.1021/jp8096583

(57) Liu, S. B. J. Phys. Chem. A 2013, 117, 962. doi: 10.1021/jp312521z

(58) Liu, S. B.; Schauer, C. K. J. Chem. Phys. 2015, 142, 054107. doi:
$10.1063 / 1.4907365$

(59) Shannon, C. E. Bell Syst. Tech. J. 1948, 27, 379. doi: 10.1002/bltj.1948.27.issue-3

(60) Sears, S. B.; Parr, R. G.; Dinur, U. Isr. J. Chem. 1980, 19, 165. doi: 10.1002/ijch.v19:1-4

(61) Sears, S. B.; Gadre, S. R. J. Chem. Phys. 1981, 75, 4626. doi: $10.1063 / 1.442578$

(62) Romera, E.; Sanchez-Moreno, P.; Dehesa, J. S. Chem. Phys. Lett. 2005, 414, 468. doi: 10.1016/j.cplett.2005.08.032

(63) Fisher, R. A. Proc. Cambridge Philos. Soc. 1925, 22, 700. doi: 10.1017/S0305004100009580

(64) Ghosh, S. K. ; Berkowitz, M.; Parr, R. G. Proc. Natl. Acad. Sci. U. S. A. 1984, 81, 8028. doi: 10.1073/pnas.81.24.8028

(65) Liu, S. B.; Rong, C. Y.; Wu, Z. M.; Lu, T. Acta Phys. -Chim. Sin. 2015, 31, 2057. [刘述斌, 荣春英, 吴泽民, 卢 天. 2015, 31, 2057.] doi: 10.3866/PKU.WHXB201509183

(66) Rényi, A. Probability Theory; North-Holland: Amsterdam, 1970.

(67) Tsallis, C. J. Stat. Phys. 1988, 52, 479. doi: 10.1007/BF01016429

(68) Onicescu, O. C. R. Acad. Sci. Paris A 1966, 263, 25.

(69) Kullback, S.; Leibler, R. A. Ann. Math. Stat. 1951, 22, 79.

(70) Parr, R. G.; Bartolotti, L. J. J. Phys. Chem. 1983, 87, 2810. doi: 10.1021/j100238a023

(71) De Proft, F.; Ayers, P. W.; Sen, K. D.; Geerlings, P. J. Chem. Phys. 2004, 120, 9969. doi: 10.1063/1.1729856

(72) Ayers, P. W. Proc. Natl. Acad. Sci. U. S. A. 2000, 97, 1959. doi: 10.1073/pnas.040539297

(73) Rong, C. Y.; Lu, T.; Liu, S. B. J. Chem. Phys. 2014, 140, 024109. doi: 10.1063/1.4860969

(74) Rong, C. Y.; Lu, T.; Chattaraj, P. K.; Liu, S. B. Indian J. Chem. Sect. A 2014, 53, 970.

(75) Becke, A. D. J. Chem. Phys. 1988, 88, 2547. doi: $10.1063 / 1.454033$

(76) Lu, T.; Chen, F. J. Comput. Chem. 2012, 33, 580. doi: $10.1002 /$ jec.v33.5

(77) von Weizsäcker, C. F. Z. Phys. 1935, 96, 431. doi: 10.1007/BF01337700

(78) March, N. H. Phys. Lett. A 1986, 113, 476. doi: 10.1016/03759601(86)90123-4

(79) Holas, A.; March, N. H. Phys. Rev. A 1991, 44, 5521. doi: 10.1103/PhysRevA.44.5521

(80) Nagy, Á. J. Chem. Phys. 2003, 119, 9401. doi: $10.1063 / 1.1615765$

(81) Flores, J. A.; Keller, J. Phys. Rev. A 1992, 45, 6259. doi: 10.1103/PhysRevA.45.6259

(82) Levy, M.; Perdew, J. P. Phys. Rev. A 1985, 32, 2010. doi: 10.1103/PhysRevA.32.2010

(83) Liu, S. B.; Parr, R. G. Phys. Rev. A 1996, 53, 2211. doi: 10.1103/PhysRevA.53.2211

(84) Liu, S. B. Phys. Rev. A 1996, 54, 1328. doi: 10.1103/PhysRevA.54.1328

(85) Borgoo, A.; Teale, A. M.; Tozer, D. J. Phys. Chem. Chem. Phys. 2015, 16, 14578. 
(86) Borgoo, A.; Tozer, D. J. J. Chem. Theory Comput. 2013, 9, 2250. doi: $10.1021 / \mathrm{ct} 400129 \mathrm{~d}$

(87) Weisskopf, V. F. Science 1975, 187, 605. doi: 10.1126/science.187.4177.605

(88) Badenhoop, J. K.; Weinhold, F. J. Chem. Phys. 1997, 107, 5406. doi: $10.1063 / 1.474248$

(89) Swain, C. G.; Scott, C. B. J. Am. Chem. Soc. 1953, 75, 141. doi: 10.1021/ja01097a041

(90) Ritchie, C. D. Accounts Chem. Res. 1972, 5, 348. doi: 10.1021/ar50058a005

(91) Mayr, H.; Patz, M. Angew. Chem. Int. Edit. 1994, 33, 938.

(92) Mayr, H.; Bug, T.; Gotta, M. F.; Hering, N.; Irrgang, B.; Janker, B.; Kempf, B.; Loos, R.; Ofial, A. R.; Remennikov, G.;
Schimmel, H. J. Am. Chem. Soc. 2001, 123, 9500. doi: 10.1021/ja010890y

(93) Lucius, R.; Loos, R.; Mayr, H. Angew. Chem. Int. Edit. 2002, 41, 91. doi: 10.1002/1521-3773(20020104)41:1<>1.0.CO;2-5

(94) Mayr, H.; Kempf, B.; Ofial, A. R. Accounts Chem. Res. 2003, 36, 66. doi: $10.1021 / a r 020094 c$

(95) Crum Brown, A.; Gibson, J. J. Chem. Soc. Trans. 1892, 61, 367. doi: $10.1039 / \mathrm{ct} 8926100367$

(96) Solomons, T. W. G.; Fryhle, C. B.; Snydeer, S. A. Organic Chemistry; Wiley: New York, USA, 2013.

(97) Wade, L. G., Jr. Organic Chemistry; Prentice-Hall: New Jersey, USA, 2003. 